\title{
Arctium Species Secondary Metabolites Chemodiversity and Bioactivities
}

\author{
Dongdong Wang 1,2,3*t, Alexandru Sabin Bădărau ${ }^{4 \dagger}$, Mallappa Kumara Swamy ${ }^{5}$, \\ Subrata Shaw ${ }^{6}$, Filippo Maggi ${ }^{7}$, Luiz Everson da Silva ${ }^{8}$, Víctor López ${ }^{9,10}$, \\ Andy Wai Kan Yeung ${ }^{11}$, Andrei Mocan ${ }^{12,13 *}$ and Atanas G. Atanasov' $2,3,14 *$
}

\section{OPEN ACCESS}

Edited by:

Helen Skaltsa,

National and Kapodistrian University

of Athens, Greece

Reviewed by:

Hiroshi Noguchi,

Nihon Pharmaceutical University,

Japan

Nishikant Wase

University of Nebraska-Lincoln,

United States

*Correspondence:

Dongdong Wang

dongdong.wang@univie.ac.at

Andrei Mocan

mocan.andrei@umfcluj.ro

Atanas G. Atanasov

atanas.atanasov@univie.ac.at

†These authors have contributed equally to this work as co-first authors

Specialty section: This article was submitted to

Plant Metabolism and Chemodiversity,

a section of the journal Frontiers in Plant Science

Received: 27 November 2018

Accepted: 11 June 2019

Published: 09 July 2019

Citation:

Wang $D$, Bădărau AS, Swamy MK, Shaw S, Maggi F, da Silva LE, López V, Yeung AWK, Mocan A and Atanasov AG (2019)

Arctium Species Secondary

Metabolites Chemodiversity and Bioactivities.

Front. Plant Sci. 10:834.

doi: 10.3389/fpls.2019.00834
${ }^{1}$ The Second Affiliated Hospital of Guizhou University of Traditional Chinese Medicine, Guiyang, China, ${ }^{2}$ Department of Molecular Biology, Institute of Genetics and Animal Breeding of the Polish Academy of Sciences, Jastrzębiec, Poland, ${ }^{3}$ Department of Pharmacognosy, Faculty of Life Sciences, University of Vienna, Vienna, Austria, ${ }^{4}$ Department of Environmental Science, Faculty of Environmental Science and Engineering, Babeş-Bolyai University, Cluj-Napoca, Romania, ${ }^{5}$ Department of Biotechnology, East West First Grade College of Science, Bengaluru, India, ${ }^{6}$ Center for the Development of Therapeutics, Broad Institute of MIT and Harvard, Cambridge, MA, United States, ${ }^{7}$ School of Pharmacy, University of Camerino, Camerino, Italy, ${ }^{8}$ Postgraduate Program in Sustainable Territorial Development, Federal University of Paraná, Curitiba, Brazil, ${ }^{9}$ Department of Pharmacy, Faculty of Health Sciences, Universidad San Jorge, Villanueva de Gállego, Spain, ${ }^{10}$ Instituto Agroalimentario de Aragón-IA2, CITA-Universidad de Zaragoza, Zaragoza, Spain, ${ }^{11}$ Oral and Maxillofacial Radiology, Applied Oral Sciences, Faculty of Dentistry, The University of Hong Kong, Hong Kong, China, ${ }^{12}$ Department of Pharmaceutical Botany, Faculty of Pharmacy, "Iuliu Haţieganu" University of Medicine and Pharmacy, Cluj-Napoca, Romania, ${ }^{13}$ Laboratory of Chromatography, Institute of Advanced Horticulture Research of Transylvania, University of Agricultural Sciences and Veterinary Medicine, Cluj-Napoca, Romania, ${ }^{14}$ Institute of Neurobiology, Bulgarian Academy of Sciences, Sofia, Bulgaria

Arctium species are known for a variety of pharmacological effects due to their diverse volatile and non-volatile secondary metabolites. Representatives of Arctium species contain non-volatile compounds including lignans, fatty acids, acetylenic compounds, phytosterols, polysaccharides, caffeoylquinic acid derivatives, flavonoids, terpenes/terpenoids and volatile compounds such as hydrocarbons, aldehydes, methoxypyrazines, carboxylic and fatty acids, monoterpenes and sesquiterpenes. Arctium species also possess bioactive properties such as anti-cancer, anti-diabetic, anti-oxidant, hepatoprotective, gastroprotective, antibacterial, antiviral, antimicrobial, anti-allergic, and anti-inflammatory effects. This review aims to provide a complete overview of the chemistry and biological activities of the secondary metabolites found in therapeutically used Arctium species. Summary of pharmacopeias and monographs contents indicating the relevant phytochemicals and therapeutic effects are also discussed, along with possible safety considerations.

Keywords: Arctium species, secondary metabolites, volatile compounds, non-volatile compounds, chemodiversity, bioactivity

\section{INTRODUCTION}

\section{Botanical and Ethnobotanical Aspects}

The genus Arctium L. (Asteraceae/Compositae, tribe Cardueae, subtribe Carduinae), together with the related genera Cousinia Cass., Hypacanthium Juz. and Schmalhausenia C. Winkl, forms the so-called Arctium-Cousinia group (de Souza et al., 2004). The species of the Arctium genus, also known as 'burdock', comprise biennial herbs occurring in waste places, streams and roadsides, less 
often in wood and forests, in temperate regions of Europe and Asia and sporadically in subtropical and tropical regions (European Scientific Cooperative on Phytotherapy, 2003). In North and South America, the genus is considered as naturalized, whereas in Africa it is quite rare. The name of the genus comes from the Greek 'arcteion' which means 'bear, alluding to the plant habitus characterized by pronounced hairiness.

According to the Plant List ${ }^{1}$, this genus encompasses 18 recognized species among which five are considered as hybrid species due to the frequent outbreeding occurring between its allogamous representatives (Lopez-Vinyallonga et al., 2010).

Arctium species are represented by hemicryptophyte plants equipped with a stout, erect taproot and entire (sporadically as dentate), rough, unarmed, alternate, tomentose, and cordate leaves. The stem is usually stout, erect, grooved, branched, and reddish. Inflorescences are formed by solitary or corymbose ovoid-conical to spherical capitula equipped with involucres made up of bracts ending with hooked apices. Receptacles are composed of numerous, hard scales. Florets are only tubulose, hermaphrodite, purple or white. Pollination is allowed by insects, mostly belonging to Lepidoptera. Fruits are having oblong, rugose achenes equipped with a golden-yellow pappus (European Scientific Cooperative on Phytotherapy, 2003).

The Arctium genus is highly polymorphic due to variability occurring in hairiness of leaves and capitula, length of floral peduncles, and color of capitula and florets. As a consequence, a sharp distinction between its members cannot occasionally be defined. In the Euro-Mediterranean area, six main species are found: A. atlanticum (Pomel) H. Lindb., A. lappa L., A. minus (Hill) Bernh., A. nemorosum Lej., A. palladini (Marcow) R.E.Fr. \& Soderb. and Arctium tomentosum Mill. (European Scientific Cooperative on Phytotherapy, 2003; Figure 1).

Arctium lappa enjoys a longstanding use in the traditional medicine (mainly roots, and, to a lesser extent, leaves and seeds) due to the bioactive properties of its metabolites (Zhao et al., 2014). To the best of our knowledge, the most utilized species for therapeutic purposes, are on first place A. lappa, also known as 'greater burdock', and, to a minor extent, $A$. minus (lesser burdock) and A. tomentosum (wooly burdock).

Arctium lappa is an herbaceous biennial plant up to $150 \mathrm{~cm}$ tall, with pubescent to subglabrous epigeal parts. Basal leaves are up to $50 \mathrm{~cm}$ in diameter, ovate, cordate and with hollow petioles. Stems are branched and end with corymbose capitula. Florets are as long as the involucral bracts. A. minus differs for the shape of inflorescence (solitary terminal capitula), dimensions of involucral bracts and capitula (smaller and shorter, respectively) and consistency of petiole (hollow). Furthermore, the bracts show a relatively hinted hairiness. A. tomentosum is characterized by petioles and peduncles covered with wooly tufts; petioles are solid. The involucral bracts are similar in dimensions to those of $A$. minus, but they show a dense covering of hairs. Florets are longer than bracts as in A. minus (European Scientific Cooperative on Phytotherapy, 2003). These three species are quite common in central Europe where often they undergo interspecific

${ }^{1}$ http://www.theplantlist.org
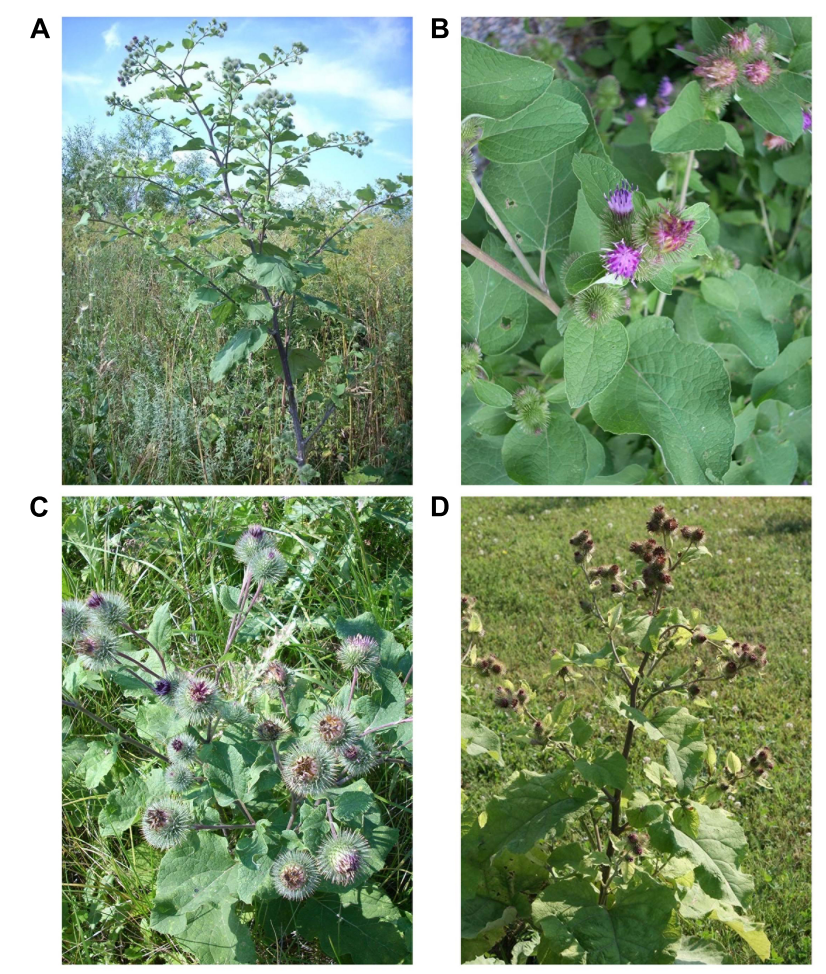

FIGURE 1 | Plants of Arctium. (A) Arctium lappa; (B) Arctium minus;

(C) Arctium tomentosum; (D) Arctium nemorosum.

hybridization giving rise to questions about their integrity (European Scientific Cooperative on Phytotherapy, 2003).

Greater burdock ( $A$. lappa) has been traditionally used in both Asian and European medicines as depurative, diuretic, carminative, anti-inflammatory, and anti-tubercular agent (Zhao et al., 2014). For therapeutic purposes, its different parts such as roots, fruits, and leaves are used. The latter have been used to treat ulcers and fester wounds (Jaric et al., 2007). They are also applied externally on the forehead to cure headache and fever, on the scalp to treat bruises and hair loss, mixed with oil and honey and applied on the chest to heal cough. In addition, under infusion, they are taken orally to treat enuresis in children (Pieroni et al., 2011). Fruits of burdock (Arctii fructus) are used to purify the blood (Lans and Turner, 2011) and to treat respiratory and infectious diseases (Bai et al., 2016). In addition, A. lappa roots, together with aerial parts of Rumex acetosella L., leaves of Ulmus rubra Muhl. and rhizomes of Rheum officinale Baill., are used to make 'Essiac', a tea used by the Ojibwa tribe of Canada for the treatment of cancer (Leonard et al., 2006). In the veterinary medicine, the root is used to treat mastitis (Lans et al., 2007), whereas the whole plant is applied against endoparasites in poultry (Lans and Turner, 2011). Besides therapeutic uses, A. lappa is also appreciated as an edible plant. For the latter purpose, young leaves, and stalks are eaten raw or cooked (Pieroni et al., 2011).

Lesser burdock (A. minus) leaves are traditionally used externally to treat rheumatic pains, fever, sunstroke, wounds, 
general infections, skin and body inflammations, alopecia, and bladder diseases (de Souza et al., 2004; Erdemoglu et al., 2009; Neves et al., 2009). They are also disposed above the body of the patient, wetted with vinegar or milk, to stimulate sweating (Sezik et al., 2001). Roots and leaves, under infusion, are also used against snake and scorpion bites and to purify the blood (Mosaddegh et al., 2012). Basal leaves and stems are also eaten raw as a snack or stewed (Tardio et al., 2005). Due to their bitter taste, they are also used to stimulate the appetite and liver functions (Tardio et al., 2005).

Wooly burdock (A. tomentosum) leaves are used as vulnerary, to treat skin rash, ulcers, abscesses, mouth sores and against rheumatic pains, whereas root is applied against alopecia and to wash hairs (Sezik et al., 2004; Saric-Kundalic et al., 2010). Roots are also employed to make a tea used for digestive problems, ulcers, rheumatisms, to purify the blood and increase sweating and as diuretic (Saric-Kundalic et al., 2010).

\section{Medicinal Uses of Arctium Species in Pharmacopeias and Monographs}

Burdock species and in particular A. lappa are used in traditional medicine for different purposes. The main traditional use of the roots of $A$. lappa in Europe comprises treatment of dermatological disorders (Saric-Kundalic et al., 2010; Miglani and Manchanda, 2014) whereas in other Eastern and Asian countries A. lappa fruits and roots are used as an antidiabetic remedy (Tousch et al., 2014; Xu et al., 2014, 2015; Ahangarpour et al., 2017). In Traditional Chinese Medicine (TCM), apart from the antidiabetic activity, the roots of $A$. lappa are considered as a blood detoxifying agent (Qin et al., 2014). In the Japanese pharmacopeia, the fruit is included as a traditional herbal medicine with recent studies revealing the potential of its extracts in oncology (Ikeda et al., 2016). The leaves of A. lappa have also been reported as an anti-inflammatory agent to relieve gastrointestinal disorders in Brazilian traditional medicine (de Almeida et al., 2013).

According to international institutions that work in the validation of traditional herbal medicines, such as the European Medicines Agency (EMA) and the European Scientific Cooperative on Phytotherapy (ESCOP), A. lappa is recommended and approved for different indications. For example, the EMA monograph approves the use of roots of A. lappa, A. minus, and A. tomentosum as an adjuvant in minor urinary tract complaints, in temporary loss of appetite and for seborrheic skin conditions (European Medicines Agency, 2011). All these indications are based upon long-standing use. In 2016, ESCOP released a monograph where the roots of all the former three species are indicated to be internally and externally used for seborrheic skin, eczema, furuncles, acne, psoriasis and internally for minor urinary tract disorders (European Scientific Cooperative on Phytotherapy-The Scientific Foundation for Herbal Medicinal Products, 2016). For oral and internal administration, the herbal drug can be used as an infusion, extract, tincture or decoction but the fresh pulp of the roots or a decoction can also be directly applied to the skin. The later monograph reveals that A. lappa preparations should not be ingested during pregnancy, lactation, or in case of hypersensitivity to the Compositae and in patients with oedema due to impaired heart or kidney function. Although certain preclinical studies can be found in the literature, clinical trials are not available for these indications approved by ESCOP and EMA.

\section{PHYTOCHEMISTRY}

\section{Non-volatile Compounds}

Till date, more than two hundred non-volatile compounds have been isolated from Arctium genus. These chemical compounds include lignans, terpenoids, sterols, flavonoids, phenolics, lactones, polyacetylenes, quinic acids, and sugars (polysaccharides). In particular, lignans are the most characteristic components in the Arctium genus. The details of chemical compounds, their occurrence in different plant parts, and the analytical methods used for their quali-quantitative determinations are briefly summarized in Table $\mathbf{1}$ whereas their description is provided in this section. The chemical structures of some compounds from Arctium species are shown in Figure 2.

\section{Lignans}

Major biologically active lignans include mainly arctigenin (a dietary phytoestrogen) and its glycoside, arctiin (lignanolides) occurring commonly in seeds, roots, fruits, and leaves of A. lappa and A. tomentosum (Yu et al., 2003; Ming et al., 2004; Liu et al., 2005, 2012, 2015; Wang et al., 2005; Matsumoto et al., 2006; Gao et al., 2008; Boldizsar et al., 2010; Ferracane et al., 2010; Zhou et al., 2011; Qin et al., 2014; Su et al., 2015; Lou et al., 2016). In addition, seeds and roots are distributed with low levels of dilignans and sesquilignans. For the first time, two new sesquilignans, namely lappaol A and B were isolated and characterized from A. lappa seeds (Ichihara et al., 1976). Later, 3 more sesquilignans, namely, lappaol C, $\mathrm{D}$, and $\mathrm{E}$, and two dilignans, namely lappaol $\mathrm{F}$ and $\mathrm{H}$, were structurally determined from the seeds of A. lappa (Ichihara et al., 1977, 1978; Yong et al., 2007; Su et al., 2015). Lappaol $\mathrm{A}, \mathrm{C}$, and $\mathrm{F}$ are also found in the fruits of A. tomentosum (Kardosova et al., 2003). Two new lignans, neoarctin A and $\mathrm{B}$, along with other recognized compounds including arctiin, arctigenin, daucosterol, lappaol F, isolappaol $\mathrm{C}$ and matairesinol were identified in seeds of A. lappa (Wang and Yang, 1995; Kardosova et al., 2003; Yong et al., 2007; Gao et al., 2008; Qin et al., 2014; Su et al., 2015). A simple RPHPLC method was developed to identify the presence of arctiin in fruits of A. lappa (Yu et al., 2003; Boldizsar et al., 2010). Using bioactivity-guided fractionation, lappaol A, C and $\mathrm{F}$, arctiin and arctignan $\mathrm{E}$ were isolated and characterized from the ethanolic extract (95\%) of A. lappa seeds (Ming et al., 2004). Likewise, HPLC/UPLC/LC/MS/MS methods have been developed to identify arctigenin and arctiin in the seeds, leaves and roots of A. lappa (Yu et al., 2003; Liu et al., 2005; Ferracane et al., 2010; Lou et al., 2010a,b; Predes et al., 2011). Further, a supercritical fluid extraction procedure was found to be superior for extracting arctiin from A. lappa 
TABLE 1 | The known non-volatile constituents of Arctium species.

\begin{tabular}{|c|c|c|c|c|c|c|}
\hline No & Compound name & Formula & Species & Plant origin/part & Analytical method & References \\
\hline & Lignans & & & & & \\
\hline 1 & Diarctigenin & $\mathrm{C}_{42} \mathrm{H}_{46} \mathrm{O}_{12}$ & A. lappa & Fruits, roots, seeds & IR/NMR/MS/TLC & $\begin{array}{l}\text { Han et al., 1994; Park et al., 2007; Qin } \\
\text { et al., } 2014\end{array}$ \\
\hline 2 & Arctiin & $\mathrm{C}_{27} \mathrm{H}_{34} \mathrm{O}_{11}$ & A. lappa, A. tomentosum & $\begin{array}{l}\text { Leaves, fruits, } \\
\text { roots, seeds }\end{array}$ & $\begin{array}{l}\text { UV/R/MS/NMR/HPLC/LCMS/ } \\
\text { MALDI-QIT-TOF MS }\end{array}$ & $\begin{array}{l}\text { Wang and Yang, 1993; Ting-Guo et al., } \\
\text { 2001; Yu et al., 2003; Ming et al., 2004; } \\
\text { Liu et al., 2005, 2012, 2015; Wang } \\
\text { et al., 2005; Matsumoto et al., 2006; } \\
\text { Boldizsar et al., 2010; Ferracane et al., } \\
\text { 2010; Zhou et al., 2011; Qin et al., } \\
\text { 2014; Su et al., 2015; Lou et al., 2016; } \\
\text { Al-Shammaa et al., } 2017\end{array}$ \\
\hline 3 & Arctigenin & $\mathrm{C}_{12} \mathrm{H}_{24} \mathrm{O}_{7}$ & A. lappa, A. tomentosum & $\begin{array}{l}\text { Leaves, fruits, } \\
\text { seeds, roots }\end{array}$ & $\begin{array}{l}\text { UV/MS/NMR/HPLC/LCMS/ } \\
\text { MALDI-QIT-TOF MS/ } \\
\text { HRESI-MS }\end{array}$ & $\begin{array}{l}\text { Umehara et al., 1993; Wang and Yang, } \\
\text { 1993; Liu et al., 2005, 2012, 2015; } \\
\text { Matsumoto et al., 2006; Gao et al., } \\
\text { 2008; Boldizsar et al., 2010; Ferracane } \\
\text { et al., 2010; Predes et al., 2011; Zhou } \\
\text { et al., 2011; Qin et al., 2014; Su et al., } \\
\text { 2015; Al-Shammaa et al., } 2017\end{array}$ \\
\hline 4 & $\begin{array}{l}\text { Arctigenin-4-O- } \beta-D- \\
\text { gentiobioside }\end{array}$ & $\mathrm{C}_{18} \mathrm{H}_{32} \mathrm{O}_{16}$ & A. lappa & Fruits & NMR/UV/IR/ORD/HRESIMS & Yang et al., 2015 \\
\hline 5 & $\begin{array}{l}\text { Arctigenin-4-O- } \alpha \text {-D- } \\
\text { galactopyranosyl-(1 } \rightarrow 6)-O-\beta \text {-D- } \\
\text { glucopyranoside }\end{array}$ & $\mathrm{C}_{18} \mathrm{H}_{32} \mathrm{O}_{16}$ & A. lappa & Fruits & NMR/UV/IR/ORD/HRESIMS & Yang et al., 2015 \\
\hline 6 & $\begin{array}{l}\text { Arctigenin-4-O- } \beta \text {-D- } \\
\text { apiofuranosyl-( }(1 \rightarrow 6)-O-\beta \text {-D- } \\
\text { glucopyranoside }\end{array}$ & $\mathrm{C}_{32} \mathrm{H}_{42} \mathrm{O}_{15}$ & A. lappa & Fruits & NMR/UV/IR/ORD/HRESIMS & Yang et al., 2015 \\
\hline 7 & $\begin{array}{l}\text { 3-benzyl-6-(1-hydroxyethyl)- } \\
\text { 2,5-piperazinedione }\end{array}$ & $\mathrm{C}_{13} \mathrm{H}_{16} \mathrm{~N}_{2} \mathrm{O}_{3}$ & A. lappa & Fruits & $\begin{array}{l}\text { IR/HR-ESI- } \\
\text { MS/NMR/CD }\end{array}$ & Yang et al., 2012 \\
\hline 8 & 3-benzyl-2,5- piperazinedione & $\mathrm{C}_{13} \mathrm{H}_{16} \mathrm{~N}_{2} \mathrm{O}_{2}$ & A. lappa & Fruits & $\begin{array}{l}\text { IR/HR-ESI- } \\
\mathrm{MS} / \mathrm{NMR} / \mathrm{CD}\end{array}$ & Yang et al., 2012 \\
\hline 9 & 5'-propanediolmatairesinoside & $\mathrm{C}_{29} \mathrm{H}_{38} \mathrm{O}_{13}$ & A. lappa & Fruits & NMR/UV/IR/ORD/HRESIMS & Yang et al., 2015 \\
\hline 10 & $\begin{array}{l}\left(7^{\prime} R, 8 R, 8^{\prime} R\right)- \\
\text { rafanotrachelogenin-4-O- } \beta \text {-D- } \\
\text { glucopyranoside }\end{array}$ & $\mathrm{C}_{27} \mathrm{H}_{34} \mathrm{O}_{12}$ & A. lappa & Fruits & NMR/UV/IR/ORD/HRESIMS & Yang et al., 2015 \\
\hline 11 & $\begin{array}{l}\left(7^{\prime} S, 8 R, 8^{\prime} R\right)- \\
\text { rafanotrachelogenin-4-O- } \beta-D- \\
\text { glucopyranoside }\end{array}$ & $\mathrm{C}_{27} \mathrm{H}_{34} \mathrm{O}_{12}$ & A. lappa & Fruits & NMR/UV/IR/ORD/HRESIMS & Yang et al., 2015 \\
\hline 12 & $\begin{array}{l}\text { (7S,8S,8'R)-4,7-dihydroxy- } \\
\text { 3,3',4-trimethoxyl-9-oxo } \\
\text { benzylbutyrolactone lignan-4- } \\
\text { O- } \beta \text {-D-glucopyranoside }\end{array}$ & $\mathrm{C}_{27} \mathrm{H}_{34} \mathrm{O}_{12}$ & A. lappa & Fruits & NMR/UV/IR/ORD/HRESIMS & Yang et al., 2015 \\
\hline
\end{tabular}


TABLE 1 | Continued

\begin{tabular}{|c|c|c|c|c|c|c|}
\hline No & Compound name & Formula & Species & Plant origin/part & Analytical method & References \\
\hline 13 & $\begin{array}{l}\left(7 \mathrm{~S}, 8 \mathrm{~S}, 8^{\prime} \mathrm{R}\right)-4,7 \text { - dihydroxy- } \\
3,3^{\prime}, 4^{\prime} \text {-trimethoxyl-9-oxo } \\
\text { dibenzylbutyrolactone lignin }\end{array}$ & $\mathrm{C}_{21} \mathrm{H}_{24} \mathrm{O}_{7}$ & A. lappa & Fruits & NMR/UV/IR/ORD/HRESIMS & Yang et al., 2015 \\
\hline 14 & $\begin{array}{l}\left(7 \mathrm{R}, 8 \mathrm{~S}, 8^{\prime} \mathrm{R}\right)-4,7,4^{\prime} \text {-trihydroxy- } \\
3,3^{\prime} \text { - dimethoxyl-9-oxo } \\
\text { dibenzylbutyrolactone lignan-4- } \\
\text { O- } \beta \text {-D-glucopyranoside }\end{array}$ & $\mathrm{C}_{26} \mathrm{H}_{32} \mathrm{O}_{12}$ & A. lappa & Fruits & NMR/UV/IR/ORD/HRESIMS & Yang et al., 2015 \\
\hline 15 & 7,8-didehydroarctigenin & $\mathrm{C} 21 \mathrm{H} 22 \mathrm{O} 5$ & A. lappa & Fruits & HRFAB/EIMS/NMR & Matsumoto et al., 2006 \\
\hline 16 & Arctiidilactone & $\mathrm{C} 2 \mathrm{OH} 20 \mathrm{O} 8$ & A. lappa & Fruits & NMR/UV/IR/ORD/HRESIMS & Yang et al., 2015 \\
\hline 17 & Arctiiapolignan A & $\mathrm{C} 2 \mathrm{OH} 28 \mathrm{O} 10$ & A. lappa & Fruits & NMR/UV/IR/ORD/HRESIMS & Yang et al., 2015 \\
\hline 18 & Arctiisesquineolignan A & $\mathrm{C} 42 \mathrm{H} 52 \mathrm{O} 19$ & A. lappa & Fruits & NMR/UV/IR/ORD/HRESIMS & Yang et al., 2015 \\
\hline 19 & Arctiisesquineolignan B & $\mathrm{C} 36 \mathrm{H} 46 \mathrm{O} 16$ & A. lappa & Fruits & UV/IR/HRESIMS/NMR & He et al., 2016 \\
\hline 20 & Arctiiphenolglycoside A & $\mathrm{C}_{19} \mathrm{H}_{28} \mathrm{O}_{13}$ & A. lappa & Fruits & UV/IR/HRESIMS/NMR & He et al., 2016 \\
\hline 21 & Arctignan A & $\mathrm{C}_{30} \mathrm{H}_{34} \mathrm{O}_{10}$ & A. lappa & Seeds & UV/MS/NMR/HPLC & Umehara et al., 1993 \\
\hline 22 & Arctignan B & $\mathrm{C}_{30} \mathrm{H}_{34} \mathrm{O}_{10}$ & A. lappa & Seeds & UV/MS/NMR/HPLC & Umehara et al., 1993 \\
\hline 23 & Arctignan C & $\mathrm{C}_{30} \mathrm{H}_{32} \mathrm{O}_{10}$ & A. lappa & Seeds & UV/MS/NMR/HPLC & Umehara et al., 1993 \\
\hline 24 & Arctignan D & $\mathrm{C}_{30} \mathrm{H}_{34} \mathrm{O}_{10}$ & A. lappa & Seeds & $\begin{array}{l}\text { UV/MS/NMR/HPLC/LCMS/ } \\
\text { MALDI-QIT-TOF MS }\end{array}$ & Umehara et al., 1993; Liu et al., 2012 \\
\hline 25 & Arctignan E & $\mathrm{C}_{40} \mathrm{H}_{44} \mathrm{O}_{13}$ & A. lappa & Seeds & UV/IR/MS/NMR/HPLC & $\begin{array}{l}\text { Umehara et al., 1993; Ming et al., 2004; } \\
\text { Ferracane et al., 2010; Qin et al., } 2014\end{array}$ \\
\hline 26 & Lappaol A & $\mathrm{C}_{30} \mathrm{H}_{32} \mathrm{O}_{9}$ & A. lappa, A. tomentosum & Seeds/fruits & TLC/UV/IR/MS/NMR/HPLC & $\begin{array}{l}\text { Ichihara et al., 1976; Ting-Guo et al., } \\
\text { 2001; Ming et al., 2004; Ferracane } \\
\text { et al., 2010; Liu et al., 2012; Qin et al., } \\
\text { 2014; Su et al., } 2015\end{array}$ \\
\hline 27 & Lappaol B & $\mathrm{C}_{31} \mathrm{H}_{34} \mathrm{O}_{9}$ & A. lappa & Seeds/fruits & NMR/MS/TLC/HPLC & Ichihara et al., 1976; Qin et al., 2014 \\
\hline 28 & Isolappaol C & $\mathrm{C}_{30} \mathrm{H}_{34} \mathrm{O}_{10}$ & A. lappa, A. tomentosum & Seeds/fruits & NMR/MS/TLC & $\begin{array}{l}\text { Park et al., 2007; Qin et al., 2014; Su } \\
\text { et al., } 2015\end{array}$ \\
\hline 29 & Lappaol C & $\mathrm{C}_{30} \mathrm{H}_{34} \mathrm{O}_{10}$ & A. lappa & Seeds & TLC/UV/IR/MS/NMR & $\begin{array}{l}\text { Ichihara et al., 1977; Ting-Guo et al., } \\
\text { 2001; Ming et al., 2004; Park et al., } \\
\text { 2007; Ferracane et al., 2010; Liu et al., } \\
\text { 2012; Su et al., } 2015\end{array}$ \\
\hline 30 & Lappaol D & $\mathrm{C}_{31} \mathrm{H}_{36} \mathrm{O}_{10}$ & A. lappa & Seeds & NMR/MS/TLC & Ichihara et al., 1977; Park et al., 2007 \\
\hline 31 & Lappaol E & $\mathrm{C}_{30} \mathrm{H}_{34} \mathrm{O}_{10}$ & A. lappa & Seeds & NMR/MS/TLC & Ichihara et al., 1977; Park et al., 2007 \\
\hline 32 & Lappaol F & $\mathrm{C}_{42} \mathrm{H}_{46} \mathrm{O}_{12}$ & A. lappa, A. tomentosum & Fruits, seeds & TLC/UV/IR/MS/NMR/HPLC & $\begin{array}{l}\text { Ting-Guo et al., 2001; Ming et al., } \\
\text { 2004; Park et al., 2007; Ferracane } \\
\text { et al., 2010; Qin et al., } 2014\end{array}$ \\
\hline 33 & Lappaol H & $\mathrm{C}_{40} \mathrm{H}_{46} \mathrm{O}_{14}$ & A. lappa & Seeds/fruits & $\begin{array}{l}\text { UV/MS/NMR/HPLC/LCMS/ } \\
\text { MALDI-QIT-TOF MS }\end{array}$ & Liu et al., 2012; Qin et al., 2014 \\
\hline 34 & Neoarctin A & $\mathrm{C}_{42} \mathrm{H}_{46} \mathrm{O}_{12}$ & A. lappa & Seeds & $\begin{array}{l}\text { UV, IR, 1H-NMR, } \\
\text { 13C-NMR, DEPT, } \\
\text { 2D-NMR and MS }\end{array}$ & $\begin{array}{l}\text { Wang and Yang, 1995; Yong et al., } \\
2007\end{array}$ \\
\hline
\end{tabular}




\begin{tabular}{|c|c|c|c|c|c|c|}
\hline No & Compound name & Formula & Species & Plant origin/part & Analytical method & References \\
\hline 35 & Neoarctin B & $\mathrm{C}_{42} \mathrm{H}_{46} \mathrm{O}_{12}$ & A. lappa & Seeds & $\begin{array}{l}\text { UV, IR, 1H-NMR, } \\
\text { 13C-NMR, DEPT, } \\
\text { 2D-NMR and MS }\end{array}$ & Wang and Yang, 1993 \\
\hline 36 & Matairesinoside & $\mathrm{C}_{26} \mathrm{H}_{32} \mathrm{O}_{11}$ & A. lappa & Fruits & UV/IR/HPLC & Boldizsar et al., 2010 \\
\hline 37 & Matairesinol & $\mathrm{C}_{20} \mathrm{H}_{22} \mathrm{O}_{6}$ & A. lappa & Seeds/fruits & $\begin{array}{l}\text { UV/MS/NMR/HPLC/LCMS/ } \\
\text { MALDI-QIT-TOF MS }\end{array}$ & $\begin{array}{l}\text { Wang and Yang, 1993; Boldizsar et al., } \\
\text { 2010; Ferracane et al., 2010; Liu et al., } \\
\text { 2012; Qin et al., 2014; Su et al., } 2015\end{array}$ \\
\hline 38 & $\begin{array}{l}\text { Matairesinol-4,4'-di-O- } \beta \text {-D- } \\
\text { glucopyranoside }\end{array}$ & $\mathrm{C}_{27} \mathrm{H}_{34} \mathrm{O}_{12}$ & A. lappa & Fruits & NMR/UV/IR/ORD/HRESIMS & Yang et al., 2015 \\
\hline 39 & Pinoresinol & $\mathrm{C}_{20} \mathrm{H}_{22} \mathrm{O}_{6}$ & A. lappa & Fruits & UV/IR/HPLC & Boldizsar et al., 2010 \\
\hline 40 & Phylligenin & $\mathrm{C}_{21} \mathrm{H}_{24} \mathrm{O}_{6}$ & A. lappa & Fruits & UV/IR/HPLC & Boldizsar et al., 2010 \\
\hline 41 & Styraxlignolide E & $\mathrm{C}_{26} \mathrm{H}_{32} \mathrm{O}_{11}$ & A. lappa & Fruits & NMR/UV/IR/ORD/HRESIMS & Yang et al., 2015 \\
\hline 42 & Styraxlignolide D & $\mathrm{C}_{26} \mathrm{H}_{32} \mathrm{O}_{11}$ & A. lappa & Fruits & NMR/UV/IR/ORD/HRESIMS & Yang et al., 2015 \\
\hline 43 & Syringaresinol & $\mathrm{C}_{22} \mathrm{H}_{26} \mathrm{O}_{8}$ & A. lappa & Roots & UV/IR/ESIMS/NMR & Han et al., 2013 \\
\hline 44 & $\begin{array}{l}(7 \mathrm{~S}, 8 \mathrm{R})-4,7,9,9^{\prime} \text {-tetrahydroxy- } \\
3,3^{\prime} \text {-dimethoxyl-7'-oxo-8-4'- } \\
\text { oxyneolignan-4-O- } \beta \text {-D- } \\
\text { glucopyranoside }\end{array}$ & $\mathrm{C}_{26} \mathrm{H}_{34} \mathrm{O}_{13}$ & A. lappa & Roots & $\begin{array}{l}\text { IR/HR-ESI- } \\
\text { MS/NMR/CD }\end{array}$ & Yang et al., 2012 \\
\hline 45 & 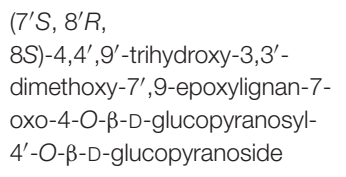 & $\mathrm{C}_{32} \mathrm{H}_{42} \mathrm{O}_{17}$ & A. lappa & Roots & $\begin{array}{l}\text { IR/HR-ESI- } \\
\text { MS/NMR/CD }\end{array}$ & Yang et al., 2012 \\
\hline 46 & $\begin{array}{l}(7 \mathrm{~S}, 8 \mathrm{R})-4,7,9,9^{\prime} \text {-tetrahydroxy- } \\
3,3^{\prime} \text { dimethoxy-8-O-4'- } \\
\text { neolignan-9'-O- } \beta \text {-D- } \\
\text { apiofuranosyl-( } 1 \rightarrow \\
\text { 6)-O- } \beta \text {-D-glucopyranoside }\end{array}$ & $\mathrm{C}_{31} \mathrm{H}_{44} \mathrm{O}_{16}$ & A. lappa & Fruits & $\begin{array}{l}\text { IR/HR-ESI- } \\
\text { MS/NMR/CD }\end{array}$ & $\begin{array}{l}\text { Huang K. et al., 2015; Huang X.Y. et al., } \\
2015\end{array}$ \\
\hline 47 & $\begin{array}{l}\text { (8R)-4,9,9'-trihydroxy-3,3'- } \\
\text { dimethoxy-7-oxo-8-O-4'- } \\
\text { neolignan-4-O- } \beta \text {-D- } \\
\text { glucopyranoside }\end{array}$ & $\mathrm{C}_{26} \mathrm{H}_{34} \mathrm{O}_{12}$ & A. lappa & Fruits & $\begin{array}{l}\text { IR/HR-ESI- } \\
\text { MS/NMR/CD }\end{array}$ & $\begin{array}{l}\text { Huang K. et al., 2015; Huang X.Y. et al., } \\
2015\end{array}$ \\
\hline 48 & $\begin{array}{l}(7 \mathrm{R}, \\
\text { 8S)-dihydrodehydrodiconiferyl } \\
\text { alcohol-7'-oxo-4-O- } \beta \text {-D- } \\
\text { glucopyranoside }\end{array}$ & $\mathrm{C}_{26} \mathrm{H}_{32} \mathrm{O}_{12}$ & A. lappa & Fruits & $\begin{array}{l}\text { IR/HR-ESI- } \\
\text { MS/NMR/CD }\end{array}$ & $\begin{array}{l}\text { Huang K. et al., 2015; Huang X.Y. et al., } \\
2015\end{array}$ \\
\hline 49 & $\begin{array}{l}\left(7^{\prime} S, 8^{\prime} R,\right. \\
\text { 8S)-4,4', } \\
\text { dimethoxy- } 7^{\prime}, 9 \text {-epoxylignan-7- } \\
\text { oxo-4-O- } \beta \text {-D-glucopyranoside }\end{array}$ & $\mathrm{C}_{26} \mathrm{H}_{32} \mathrm{O}_{12}$ & A. lappa & Fruits & $\begin{array}{l}\text { IR/HR-ESI- } \\
\text { MS/NMR/CD }\end{array}$ & $\begin{array}{l}\text { Huang K. et al., 2015; Huang X.Y. et al., } \\
2015\end{array}$ \\
\hline \multirow[t]{2}{*}{50} & Trachelogenin & $\mathrm{C}_{21} \mathrm{H}_{24} \mathrm{O}_{7}$ & A. lappa & Fruits & - & Ichikawa et al., 1986 \\
\hline & Terpenes/Terpenoids & & & & & \\
\hline 51 & $\beta$-eudesmol & $\mathrm{C}_{15} \mathrm{H}_{26} \mathrm{O}$ & A. lappa & Fruits & - & Yayli et al., 2005 \\
\hline 52 & Ursolic acid & $\mathrm{C}_{30} \mathrm{H}_{48} \mathrm{O}_{3}$ & A. lappa & Root & UV/IR/ESIMS/NMR & Han et al., 2013 \\
\hline
\end{tabular}

8S)-4, $4^{\prime}, 9^{\prime}$-trihydroxy-3,3'-

dimethoxy-7',9-epoxylignan-7-

4 '-O- $\beta-\mathrm{D}-\mathrm{g}$-glucopyranosy

(7S, 8R)-4,7,9,9'-tetrahydroxy-

S)-4,4', $9^{\prime}$-trihydroxy-3,3'- 


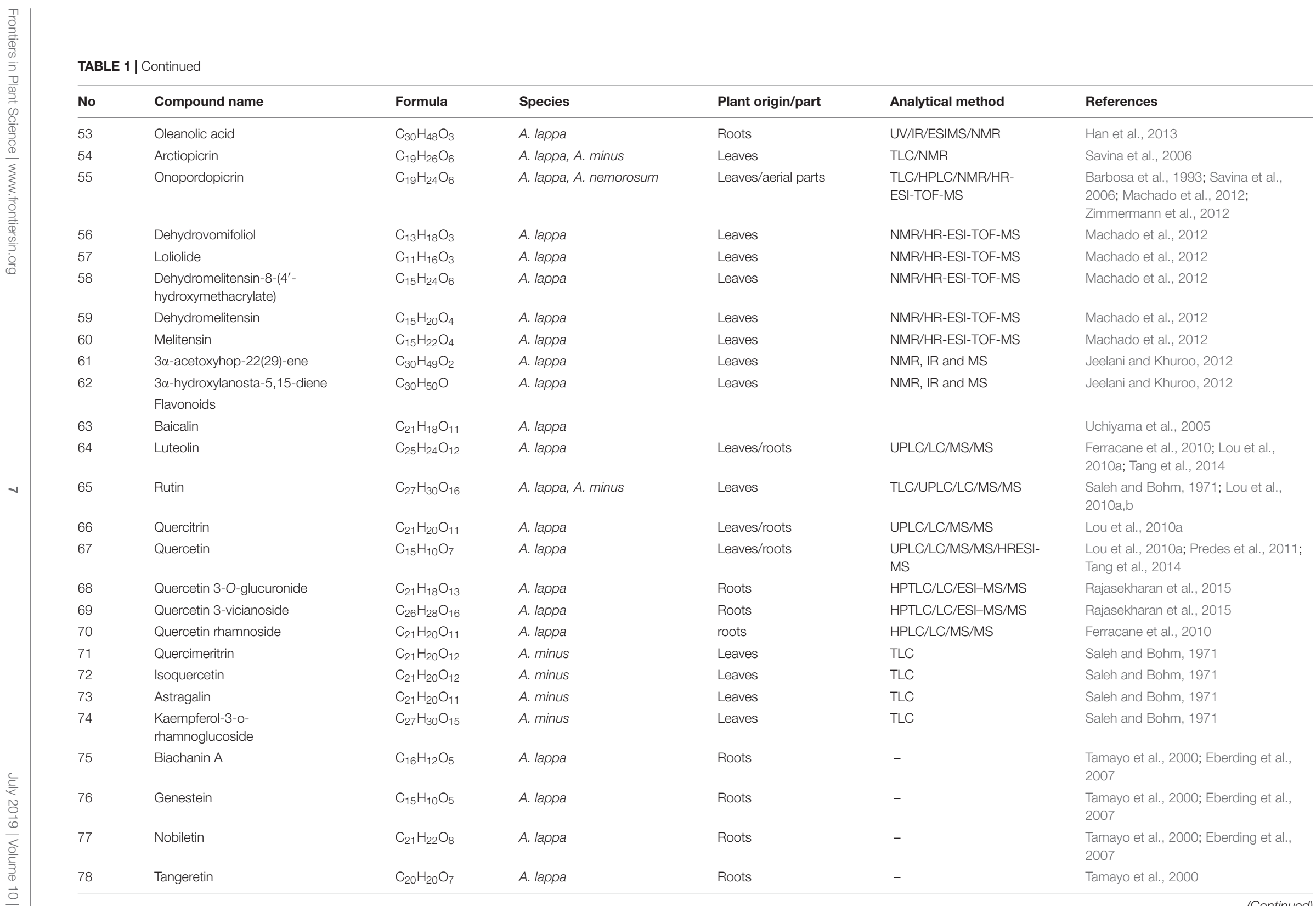




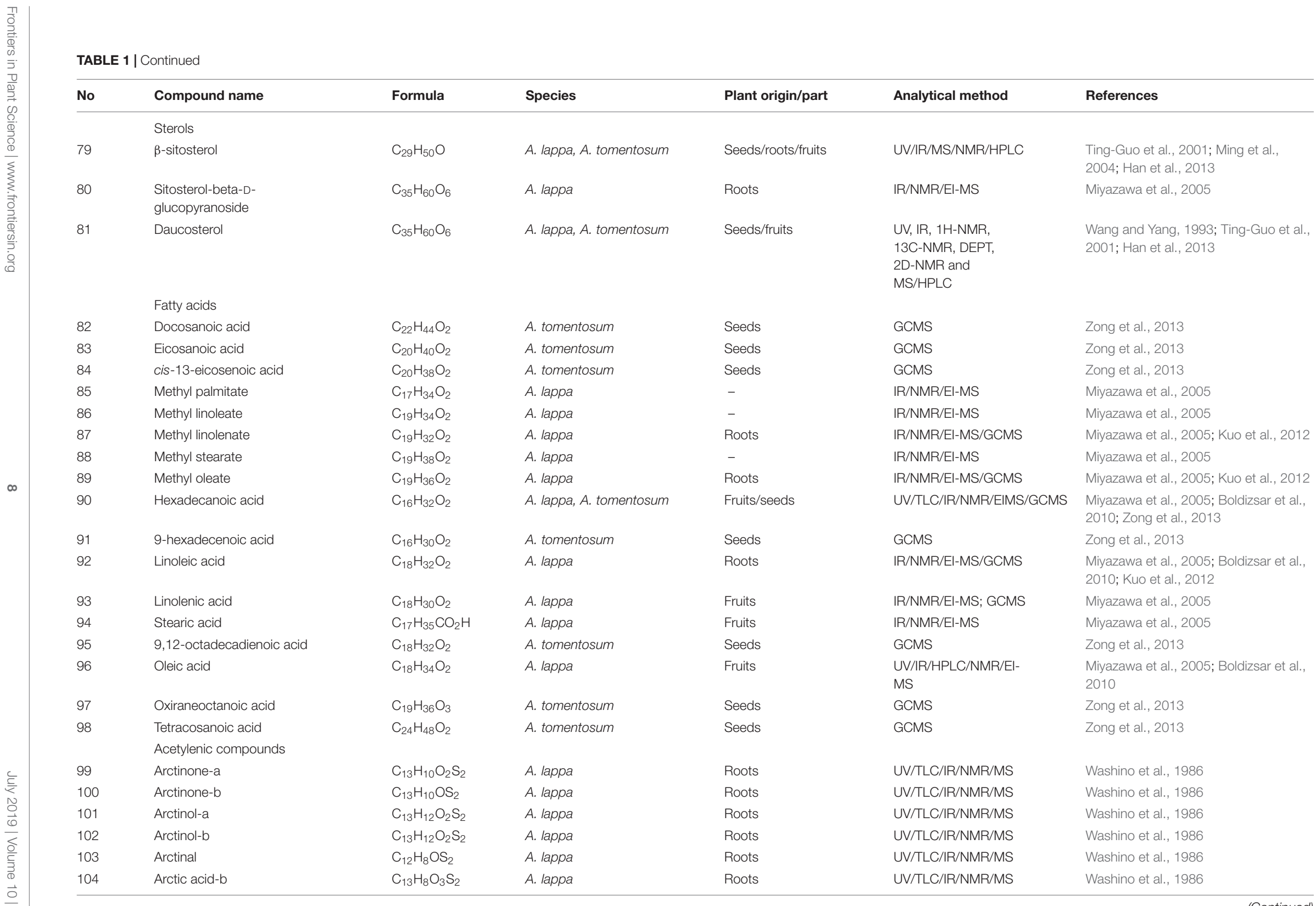


TABLE 1 | Continued

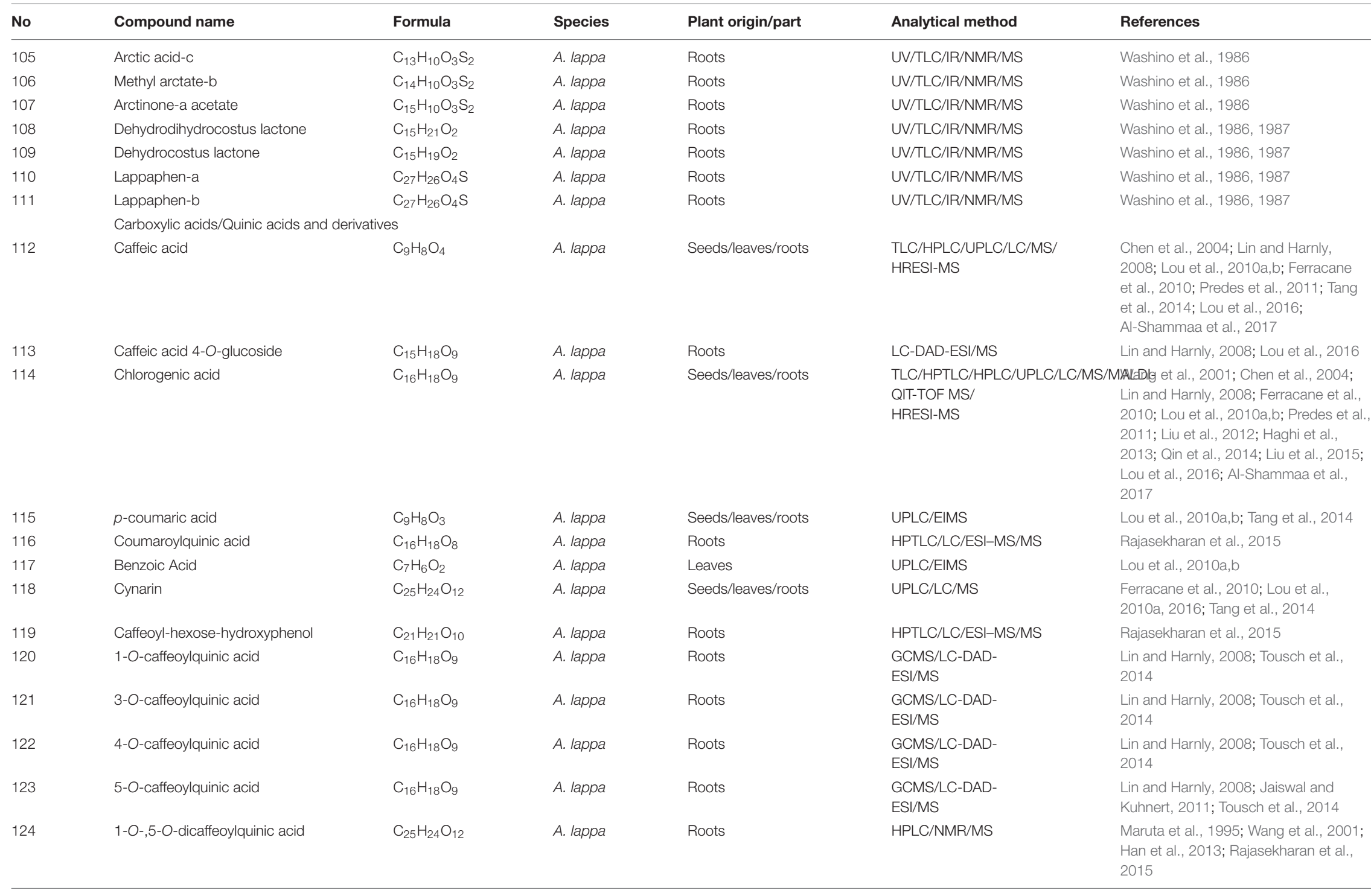


TABLE 1 | Continued

\begin{tabular}{|c|c|c|c|c|c|c|}
\hline No & Compound name & Formula & Species & Plant origin/part & Analytical method & References \\
\hline 125 & $\begin{array}{l}\text { 1-O-, 5-O-dicaffeoyl-3-O- } \\
\text { succinylquinaiccid }\end{array}$ & $\mathrm{C}_{35} \mathrm{H}_{40} \mathrm{O}_{15}$ & A. lappa & Roots & NMR/EI-MS & Maruta et al., 1995 \\
\hline 126 & $\begin{array}{l}\text { 1-O,-5-O-dicaffeoyl-4-O- } \\
\text { succinylquinic } \\
\text { acid }\end{array}$ & $\mathrm{C}_{29} \mathrm{H}_{35} \mathrm{O}_{15}$ & A. lappa & Roots & NMR/MS & Maruta et al., 1995 \\
\hline 127 & 1-O-,5-O-dicaffeoyl-3-O- & $\mathrm{C}_{33} \mathrm{H}_{39} \mathrm{O}_{18}$ & A. lappa & Roots & NMR/MS & Maruta et al., 1995 \\
\hline 128 & $\begin{array}{l}\text { 4-O-disuccinylquaicniidc and } \\
\text { 1-O-,3-0-,5-O-tricaffeoyl-4-O- } \\
\text { succinylquinic } \\
\text { acid }\end{array}$ & $\mathrm{C}_{38} \mathrm{H}_{41} \mathrm{O}_{18}$ & A. lappa & Roots & NMR/MS & Maruta et al., 1995 \\
\hline 129 & 1,3-di-O-caffeoylquinic acid & $\mathrm{C}_{25} \mathrm{H}_{24} \mathrm{O}_{12}$ & A. lappa & Seeds/roots & $\begin{array}{l}\text { LCMS/ MALDI-QIT-TOF } \\
\text { MS }\end{array}$ & Lin and Harnly, 2008; Liu et al., 2012 \\
\hline 130 & 1,5-di-O-caffeoylquinic acid & $\mathrm{C}_{25} \mathrm{H}_{24} \mathrm{O}_{12}$ & A. lappa & Leaves/Seeds/roots & $\begin{array}{l}\text { UPLC/HPLC/PDA/LCMS/ } \\
\text { MALDI-QIT-TOF MS }\end{array}$ & $\begin{array}{l}\text { Maruta et al., 1995; Lin and Harnly, } \\
\text { 2008; Liu et al., 2012; Haghi et al., } \\
\text { 2013; Tousch et al., } 2014\end{array}$ \\
\hline 131 & $\begin{array}{l}\text { 1,5-di-O-caffeoyl-4-O- } \\
\text { maloylquinic } \\
\text { acid }\end{array}$ & $\mathrm{C}_{29} \mathrm{H}_{27} \mathrm{O}_{16}$ & A. lappa & Roots & $\begin{array}{l}\text { LCMS/ MALDI-QIT-TOF } \\
\text { MS }\end{array}$ & $\begin{array}{l}\text { Jaiswal and Kuhnert, 2011; Liu et al., } \\
\text { 2012; Tousch et al., } 2014\end{array}$ \\
\hline 132 & $\begin{array}{l}\text { 1,5-di-O-caffeoyl-3-O- } \\
\text { maloylquinic } \\
\text { acid }\end{array}$ & $\mathrm{C}_{25} \mathrm{H}_{27} \mathrm{O}_{16}$ & A. lappa & Roots & $\begin{array}{l}\text { LCMS/ MALDI-QIT-TOF } \\
\text { MS }\end{array}$ & $\begin{array}{l}\text { Jaiswal and Kuhnert, 2011; Liu et al., } \\
\text { 2012; Tousch et al., } 2014\end{array}$ \\
\hline 133 & $\begin{array}{l}\text { 1,5-di-O-caffeoyl-3-O- } \\
\text { succinoylquinic } \\
\text { acid }\end{array}$ & $\mathrm{C}_{29} \mathrm{H}_{27} \mathrm{O}_{15}$ & A. lappa & Roots & $\begin{array}{l}\text { LCMS/ MALDI-QIT-TOF } \\
\text { MS }\end{array}$ & $\begin{array}{l}\text { Maruta et al., 1995; Jaiswal and } \\
\text { Kuhnert, 2011; Liu et al., 2012; Tousch } \\
\text { et al., } 2014\end{array}$ \\
\hline 134 & $\begin{array}{l}\text { 1,5-di-O-caffeoyl-3,4-di-O- } \\
\text { succinoylquinic } \\
\text { acid }\end{array}$ & $\mathrm{C}_{33} \mathrm{H}_{31} \mathrm{O}_{18}$ & A. lappa & Roots & $\begin{array}{l}\text { LCMS/ MALDI-QIT-TOF } \\
\text { MS }\end{array}$ & Liu et al., 2012; Tousch et al., 2014 \\
\hline 135 & $\begin{array}{l}\text { 1,3,5-tri-O-caffeoyl-4-O- } \\
\text { succinoylquinic } \\
\text { acid }\end{array}$ & $\mathrm{C}_{38} \mathrm{H}_{33} \mathrm{O}_{18}$ & A. lappa & Roots & $\begin{array}{l}\text { GCMS/LCMS/ } \\
\text { MALDI-QIT-TOF MS }\end{array}$ & $\begin{array}{l}\text { Jaiswal and Kuhnert, 2011; Liu et al., } \\
\text { 2012; Tousch et al., } 2014\end{array}$ \\
\hline 136 & 1,3,5-tri-O-caffeoylquinic acid & $\mathrm{C}_{34} \mathrm{H}_{29} \mathrm{O}_{15}$ & A. lappa & Roots & $\begin{array}{l}\text { GCMS/LCMS/ } \\
\text { MALDI-QIT-TOF MS }\end{array}$ & Liu et al., 2012; Tousch et al., 2014 \\
\hline 137 & $\begin{array}{l}\text { 1,5-di-O-caffeoyl-3-O- } \\
\text { succinoyl-4-O-maloyquinic } \\
\text { acid }\end{array}$ & - & A. lappa & Roots & $\begin{array}{l}\text { LCMS/ MALDI-QIT-TOF } \\
\text { MS }\end{array}$ & Liu et al., 2012 \\
\hline 138 & 5-sinapoylquinic acid & $\mathrm{C}_{18} \mathrm{H}_{22} \mathrm{O}_{10}$ & A. lappa & Roots & LC-DAD-ESI/MS & Lin and Harnly, 2008 \\
\hline 139 & 3-sinapoyl-5-caffeoylquinic acid & $\mathrm{C}_{27} \mathrm{H}_{28} \mathrm{O}_{13}$ & A. lappa & Roots & LC-DAD-ESI/MS & Lin and Harnly, 2008 \\
\hline 140 & $\begin{array}{l}\text { 3-sinapoyl-5-caffeoyl-1- } \\
\text { methoxyoxaloylquinic } \\
\text { acid }\end{array}$ & - & A. lappa & Roots & LC-DAD-ESI/MS & Lin and Harnly, 2008 \\
\hline
\end{tabular}


TABLE 1 | Continued

\begin{tabular}{|c|c|c|c|c|c|c|}
\hline No & Compound name & Formula & Species & Plant origin/part & Analytical method & References \\
\hline 141 & $\begin{array}{l}\text { 4-sinapoyl-5-caffeoyl-1- } \\
\text { methoxyoxaloylquinic } \\
\text { acid }\end{array}$ & - & A. lappa & Roots & LC-DAD-ESI/MS & Lin and Harnly, 2008 \\
\hline 142 & 3,4-dicaffeoylquinic acid & $\mathrm{C}_{25} \mathrm{H}_{24} \mathrm{O}_{12}$ & A. lappa & Roots/seeds & LC-DAD-ESI/MS & Lin and Harnly, 2008 \\
\hline 143 & 1,4-di-O-caffeoylquinic acid & $\mathrm{C}_{25} \mathrm{H}_{23} \mathrm{O}_{12}$ & A. lappa & Roots & $\begin{array}{l}\text { GCMS/LC-DAD- } \\
\text { ESI/MS }\end{array}$ & $\begin{array}{l}\text { Lin and Harnly, 2008; Jaiswal and } \\
\text { Kuhnert, 2011; Tousch et al., } 2014\end{array}$ \\
\hline 144 & 3,5-di-O-caffeoylquinic acid & $\mathrm{C}_{25} \mathrm{H}_{24} \mathrm{O}_{12}$ & A. lappa & Roots & $\begin{array}{l}\text { GCMS/LC-DAD- } \\
\text { ESI/MS }\end{array}$ & $\begin{array}{l}\text { Lin and Harnly, 2008; Jaiswal and } \\
\text { Kuhnert, 2011; Tousch et al., } 2014\end{array}$ \\
\hline 145 & 4,5-dicaffeoylquinic acid & $\mathrm{C}_{25} \mathrm{H}_{24} \mathrm{O}_{12}$ & A. lappa & Roots/seeds & LC-DAD-ESI/MS & Lin and Harnly, 2008 \\
\hline 146 & $\begin{array}{l}\text { 3,5-dicaffeoyl-1- } \\
\text { methoxyoxaloylquinic } \\
\text { acid }\end{array}$ & - & A. lappa & Roots & LC-DAD-ESI/MS & Lin and Harnly, 2008 \\
\hline 147 & 3-feruloyl-5-caffeoylquinic acid & - & A. lappa & Roots & LC-DAD-ESI/MS & Lin and Harnly, 2008 \\
\hline 148 & $\begin{array}{l}\text { 4,5-dicaffeoyl-1- } \\
\text { methoxyoxaloylquinic } \\
\text { acid }\end{array}$ & - & A. lappa & Roots & LC-DAD-ESI/MS & Lin and Harnly, 2008 \\
\hline 149 & $\begin{array}{l}\text { 3-sinapoyl-5-caffeoyl-4- } \\
\text { methoxyoxaloylquinic } \\
\text { acid }\end{array}$ & - & A. lappa & Roots & LC-DAD-ESI/MS & Lin and Harnly, 2008 \\
\hline 150 & 1,4,5-tricaffeoylquinic acid & $\mathrm{C}_{34} \mathrm{H}_{30} \mathrm{O}_{15}$ & A. lappa & Roots & LC-DAD-ESI/MS & Lin and Harnly, 2008 \\
\hline 151 & 3,4,5-tricaffeoylquinic acid & - & A. lappa & Roots & LC-DAD-ESI/MS & Lin and Harnly, 2008 \\
\hline 152 & $\begin{array}{l}\text { 1,4,5-tricaffeoyl-3- } \\
\text { methoxyoxaloylquinic } \\
\text { acid }\end{array}$ & - & A. lappa & Roots & LC-DAD-ESI/MS & Lin and Harnly, 2008 \\
\hline 153 & 3-succinoyl-4,5-dicaffeoyl & - & A. lappa & Roots & LCMS & Jaiswal and Kuhnert, 2011 \\
\hline 154 & $\begin{array}{l}\text { 1,5-dicaffeoyl-3-succinoylquinic } \\
\text { acid }\end{array}$ & - & A. lappa & Roots & HPLC/LCMS & $\begin{array}{l}\text { Wang et al., 2001; Jaiswal and } \\
\text { Kuhnert, } 2011\end{array}$ \\
\hline 155 & $\begin{array}{l}\text { 1,5-di-O-caffeoyl-4-O- } \\
\text { succinoylquinic } \\
\text { acid }\end{array}$ & $\mathrm{C}_{29} \mathrm{H}_{27} \mathrm{O}_{15}$ & A. lappa & Roots & GCMS/LCMS & $\begin{array}{l}\text { Maruta et al., 1995; Jaiswal and } \\
\text { Kuhnert, 2011; Liu et al., 2012; Tousch } \\
\text { et al., } 2014\end{array}$ \\
\hline 156 & $\begin{array}{l}\text { 3,4-dicaffeoyl-5-succinoylquinic } \\
\text { acid }\end{array}$ & $\mathrm{C}_{29} \mathrm{H}_{28} \mathrm{O}_{15}$ & A. lappa & Roots & LCMS & Jaiswal and Kuhnert, 2011 \\
\hline 157 & $\begin{array}{l}\text { 1,3-dicaffeoyl-5-fumaroylquinic } \\
\text { acid }\end{array}$ & - & A. lappa & Roots & LCMS & Jaiswal and Kuhnert, 2011 \\
\hline 158 & $\begin{array}{l}\text { 1,5-dicaffeoyl-4-fumaroylquinic } \\
\text { acid }\end{array}$ & - & A. lappa & Roots & LCMS & Jaiswal and Kuhnert, 2011 \\
\hline 159 & $\begin{array}{l}\text { 1,5-dicaffeoyl-3-maloylquinic } \\
\text { acid }\end{array}$ & - & A. lappa & Roots & LCMS & Jaiswal and Kuhnert, 2011 \\
\hline
\end{tabular}


TABLE 1 | Continued

\begin{tabular}{|c|c|c|c|c|c|c|}
\hline No & Compound name & Formula & Species & Plant origin/part & Analytical method & References \\
\hline 160 & $\begin{array}{l}\text { 1,4-di-O-caffeoyl-3-O- } \\
\text { maloylquinic } \\
\text { Acid }\end{array}$ & $\mathrm{C}_{29} \mathrm{H}_{27} \mathrm{O}_{16}$ & A. lappa & Roots & LCMS & Jaiswal and Kuhnert, 2011 \\
\hline 161 & $\begin{array}{l}\text { 1,3-di-O-caffeoyl-4,5-di-O- } \\
\text { maloylquinic }\end{array}$ & $\mathrm{C}_{33} \mathrm{H}_{31} \mathrm{O}_{20}$ & A. lappa & Roots & GCMS/LCMS & $\begin{array}{l}\text { Jaiswal and Kuhnert, 2011; Tousch } \\
\text { et al., } 2014\end{array}$ \\
\hline 162 & $\begin{array}{l}\text { 1,5-dicaffeoyl-4-maloylquinic } \\
\text { acid }\end{array}$ & - & A. lappa & Roots & LCMS & Jaiswal and Kuhnert, 2011 \\
\hline 163 & $\begin{array}{l}\text { 1,4-di-O-maloyl-3,5-di-O- } \\
\text { caffeoylquinic } \\
\text { acid }\end{array}$ & $\mathrm{C}_{31} \mathrm{H}_{33} \mathrm{O}_{20}$ & A. lappa & Roots & GCMS/LCMS & $\begin{array}{l}\text { Jaiswal and Kuhnert, 2011; Tousch } \\
\text { et al., } 2014\end{array}$ \\
\hline 164 & $\begin{array}{l}\text { 1,3,5-tricaffeoyl-4- } \\
\text { succinoylquinic } \\
\text { acid }\end{array}$ & - & A. lappa & Roots & LCMS & Jaiswal and Kuhnert, 2011 \\
\hline 165 & $\begin{array}{l}\text { 1,5-dicaffeoyl-3,4- } \\
\text { disuccinoylquinic } \\
\text { acid }\end{array}$ & - & A. lappa & Roots & LCMS & Jaiswal and Kuhnert, 2011 \\
\hline 166 & $\begin{array}{l}\text { 1,5-dicaffeoyl-3-fumaroyl-4- } \\
\text { succinoylquinic } \\
\text { acid }\end{array}$ & - & A. lappa & Roots & LCMS & Jaiswal and Kuhnert, 2011 \\
\hline 167 & $\begin{array}{l}\text { 1-fumaroyl-3,5-dicaffeoyl-4- } \\
\text { succinoylquinic } \\
\text { acid }\end{array}$ & - & A. lappa & Roots & LCMS & Jaiswal and Kuhnert, 2011 \\
\hline 168 & $\begin{array}{l}\text { 1,5-di-O-caffeoyl-3-O- } \\
\text { succinoyl-4-O-maloylquinic } \\
\text { acid }\end{array}$ & $\mathrm{C}_{33} \mathrm{H}_{31} \mathrm{O}_{19}$ & A. lappa & Roots & GCMS/LCMS & $\begin{array}{l}\text { Jaiswal and Kuhnert, 2011; Tousch } \\
\text { et al., } 2014\end{array}$ \\
\hline 169 & $\begin{array}{l}\text { Dimaloyl-dicaffeoylquinic acid } \\
\text { isomer } 1\end{array}$ & $\mathrm{C}_{33} \mathrm{H}_{31} \mathrm{O}_{20}$ & A. lappa & Roots & GCMS/LCMS & Tousch et al., 2014 \\
\hline 170 & $\begin{array}{l}\text { Succinoyl-tricaffeoylquinic acid } \\
\text { isomer }\end{array}$ & $\mathrm{C}_{38} \mathrm{H}_{33} \mathrm{O}_{18}$ & A. lappa & Roots & GCMS/LCMS & Tousch et al., 2014 \\
\hline 171 & $\begin{array}{l}\text { Maloyl-dicaffeoylquinic acid } \\
\text { isomer }\end{array}$ & $\mathrm{C}_{29} \mathrm{H}_{27} \mathrm{O}_{15}$ & A. lappa & Roots & GCMS/LCMS & Tousch et al., 2014 \\
\hline 172 & $\begin{array}{l}\text { Dicaffeoyl-succinoyl- } \\
\text { malonylquinic acid isomer } \\
1\end{array}$ & $\mathrm{C}_{33} \mathrm{H}_{31} \mathrm{O}_{19}$ & A. lappa & Roots & GCMS/LCMS & Tousch et al., 2014 \\
\hline 173 & $\begin{array}{l}\text { Dicaffeoyl-succinoyl- } \\
\text { malonylquinic acid isomer } \\
2\end{array}$ & $\mathrm{C}_{33} \mathrm{H}_{31} \mathrm{O}_{20}$ & A. lappa & Roots & GCMS/LCMS & Tousch et al., 2014 \\
\hline 174 & $\begin{array}{l}\text { Dimaloyl-dicaffeoylquinic acid } \\
\text { isomer } 2\end{array}$ & $\mathrm{C}_{33} \mathrm{H}_{31} \mathrm{O}_{20}$ & A. lappa & Roots & GCMS/LCMS & Tousch et al., 2014 \\
\hline 175 & $\begin{array}{l}\text { Dimaloyl-dicaffeoylquinic acid } \\
\text { isomer } 3\end{array}$ & $\mathrm{C}_{33} \mathrm{H}_{31} \mathrm{O}_{20}$ & A. lappa & Roots & GCMS/LCMS & Tousch et al., 2014 \\
\hline
\end{tabular}


TABLE 1 | Continued

\begin{tabular}{|c|c|c|c|c|c|c|}
\hline No & Compound name & Formula & Species & Plant origin/part & Analytical method & References \\
\hline 176 & Maloyl-tricaffeoylquinic isomer & $\mathrm{C}_{28} \mathrm{H}_{32} \mathrm{O}_{19}$ & A. lappa & Roots & GCMS/LCMS & Tousch et al., 2014 \\
\hline 177 & $\begin{array}{l}\text { 1,3,5-tri-O-caffeoyl-4-O- } \\
\text { maloylquinic } \\
\text { Acid }\end{array}$ & $\mathrm{C}_{38} \mathrm{H}_{33} \mathrm{O}_{19}$ & A. lappa & Roots & GCMS/LCMS & Tousch et al., 2014 \\
\hline 178 & 5-hydroxymaltol & $\mathrm{C}_{6} \mathrm{H}_{6} \mathrm{O}_{4}$ & A. lappa & Roots & UV/IR/ESIMS/NMR & Han et al., 2013 \\
\hline \multirow[t]{2}{*}{179} & Succinic acid & $\mathrm{C}_{4} \mathrm{H}_{6} \mathrm{O}_{4}$ & A. lappa & Roots & UV/IR/ESIMS/NMR & Han et al., 2013 \\
\hline & Saccharides/Polysaccharides & & & & & \\
\hline 180 & Rhamnogalacturonan & $\mathrm{C}_{117} \mathrm{H}_{178} \mathrm{O}_{101}$ & A. lappa, A. minus & Roots/leaves & $\begin{array}{l}\text { Chromatography/NMR/sugar } \\
\text { analysis }\end{array}$ & $\begin{array}{l}\text { Kato and Watanabe, 1993; Carlotto } \\
\text { et al., } 2016\end{array}$ \\
\hline 181 & Xylan & $\left(\mathrm{C}_{5} \mathrm{H}_{8} \mathrm{O}_{4}\right) \mathrm{n}$ & A. lappa, A. minus & Roots/leaves & $\begin{array}{l}\text { Chromatography/NMR/sugar } \\
\text { analysis }\end{array}$ & Kato and Watanabe, 1993 \\
\hline 182 & Arabinan & $\mathrm{C}_{9} \mathrm{H}_{13} \mathrm{~N}_{3} \mathrm{O}_{5}$ & A. lappa, A. minus & Roots/leaves & $\begin{array}{l}\text { Chromatography/ } \\
\text { NMR/sugar analysis }\end{array}$ & $\begin{array}{l}\text { Kato and Watanabe, 1993; Carlotto } \\
\text { et al., } 2016\end{array}$ \\
\hline 183 & Arabinogalactan & $\mathrm{C}_{20} \mathrm{H}_{36} \mathrm{O}_{14}$ & A. lappa, A. minus & Roots/leaves & $\begin{array}{l}\text { Chromatography/ } \\
\text { NMR/sugar analysis }\end{array}$ & $\begin{array}{l}\text { Kato and Watanabe, 1993; Carlotto } \\
\text { et al., } 2016\end{array}$ \\
\hline 184 & Galactan & $\mathrm{C}_{18} \mathrm{H}_{32} \mathrm{O}_{16}$ & A. lappa, A. minus & Roots/leaves & $\begin{array}{l}\text { Chromatography/ } \\
\text { NMR/sugar analysis }\end{array}$ & Kato and Watanabe, 1993 \\
\hline 185 & Cellulose & $\mathrm{C}_{64} \mathrm{H}_{124} \mathrm{O}_{30}$ & A. lappa, A. minus & Roots/leaves & $\begin{array}{l}\text { Chromatography/ } \\
\text { NMR/sugar analysis }\end{array}$ & Kato and Watanabe, 1993 \\
\hline 186 & Xyloglucan & $\mathrm{C}_{51} \mathrm{H}_{86} \mathrm{O}_{42}$ & A. lappa, A. minus & Roots/leaves & $\begin{array}{l}\text { Chromatography/ } \\
\text { NMR/sugar analysis }\end{array}$ & Kato and Watanabe, 1993 \\
\hline 187 & Galacturonic acid & $\mathrm{C}_{6} \mathrm{H}_{10} \mathrm{O}_{7}$ & A. lappa & Roots/leaves & Chromatography/ NMR & Carlotto et al., 2016 \\
\hline 188 & Galacturonic acid & $\mathrm{C}_{6} \mathrm{H}_{10} \mathrm{O}_{7}$ & A. lappa & Roots & Chromatography & Fuchigami et al., 1990 \\
\hline 189 & Galactose & $\mathrm{C}_{6} \mathrm{H}_{12} \mathrm{O}_{6}$ & A. lappa & Roots/leaves/fruits & Chromatography/ NMR & $\begin{array}{l}\text { Fuchigami et al., 1990; Kardosova } \\
\text { et al., 2003; Boldizsar et al., 2010; } \\
\text { Carlotto et al., } 2016\end{array}$ \\
\hline 190 & Glucose & $\mathrm{C}_{6} \mathrm{H}_{12} \mathrm{O}_{6}$ & A. lappa & Roots/leaves/fruits & UV/NMR/HPLC/GCMS & $\begin{array}{l}\text { Kardosova et al., 2003; Boldizsar et al., } \\
\text { 2010; Li et al., 2013; Carlotto et al., } \\
2016\end{array}$ \\
\hline 191 & Mannose & $\mathrm{C}_{6} \mathrm{H}_{12} \mathrm{O}_{6}$ & A. lappa & Roots/leaves & NMR & Carlotto et al., 2016 \\
\hline 192 & Sucrose & $\mathrm{C}_{12} \mathrm{H}_{22} \mathrm{O}_{11}$ & A. lappa & Roots & UV/NMR/HPLC/GCMS & Boldizsar et al., 2010; Li et al., 2013 \\
\hline 193 & Raffinose & $\mathrm{C}_{18} \mathrm{H}_{32} \mathrm{O}_{16}$ & A. lappa & Fruits & UV/NMR/HPLC/GCMS & Boldizsar et al., 2010 \\
\hline 194 & Rhamnose & $\mathrm{C}_{6} \mathrm{H}_{12} \mathrm{O}_{5}$ & A. lappa & Roots/leaves/fruits & UV/NMR/HPLC/GCMS & $\begin{array}{l}\text { Boldizsar et al., 2010; Carlotto et al., } \\
2016\end{array}$ \\
\hline 195 & Arabinose & $\mathrm{C}_{5} \mathrm{H}_{10} \mathrm{O}_{5}$ & A. lappa & Roots/leaves/fruits & UV/NMR/HPLC/GCMS & $\begin{array}{l}\text { Fuchigami et al., 1990; Kardosova } \\
\text { et al., 2003; Boldizsar et al., 2010; } \\
\text { Carlotto et al., } 2016\end{array}$ \\
\hline 196 & Inulin (fructan) & (C6H10O5)n & A. lappa, A. tomentosum & Roots & $\begin{array}{l}\text { HPTLC/MS/NMR/HPLC- } \\
\text { ELSD }\end{array}$ & $\begin{array}{l}\text { Kardosova et al., 2003; } \\
\text { Turdumambetov et al., 2004; Milani } \\
\text { et al., 2011; Olennikov and Tankhaeva, } \\
\text { 2011; Li et al., 2013; Liu et al., } 2014\end{array}$ \\
\hline 197 & Fructose & $\mathrm{C}_{6} \mathrm{H}_{12} \mathrm{O}_{6}$ & A. lappa & Roots & HPLC-ELSD & Li et al., 2013 \\
\hline
\end{tabular}




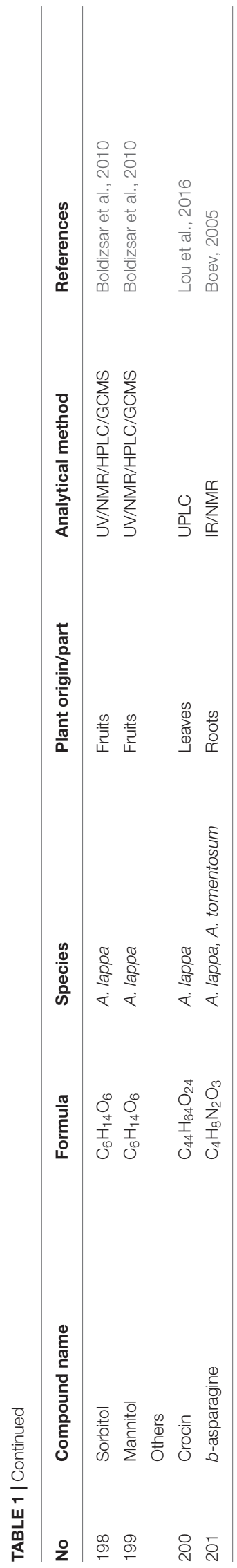

fruits (Carlotto et al., 2016). A high-speed counter-current chromatography was employed to obtain the pure compound arctiin from the fruit extracts of A. lappa. Authors obtained $49 \%$ of arctiin identified based on LC-MS and NMR techniques (Wang et al., 2005). A novel butyrolactone lignan compound named diarctigenin was found to occur in the methanolic seed extracts of A. lappa (Han et al., 1994). The fruits of A. lappa are reported to contain a total of 13 compounds including 5 new natural products (Umehara et al., 1993). Among them, 6 compounds were identified as arctignan A-E and artctigenin. Later, the occurrence of arctigenin and arctiin was also established from the leaves and seeds of $A$. lappa (Umehara et al., 1993; Liu et al., 2005; Matsumoto et al., 2006). Besides, the active extract resulted from the bioassay-guided fractionation of seed methanolic extract contained five active compounds including a new sesquilignan named isolappaol C and four known sesquilignan and dilignans namely, diarctigenin, and lappaol C, D, and F (Ferracane et al., 2010). Further, improved methods of extraction and analysis revealed that the seeds and roots of $A$. lappa contain arctigenin, arctiin, arctignan E, matareisinol, lappaol A, C, and F (Ferracane et al., 2010; Lou et al., 2010a, 2016; Liu et al., 2015; Su et al., 2015). The occurrence of 8 lignans in seeds and 1 lignan in roots of $A$. lappa was determined. The identified lignans were arctiin, arctigenin arctignan $\mathrm{D}$ and $\mathrm{E}$, lappaol $\mathrm{A}, \mathrm{C}$, and $\mathrm{H}$, isolappaol C and matairesinol (Liu et al., 2012; Haghi et al., 2013). Likewise, syringaresinol was reported in the chloroform fraction of A. lappa roots (Han et al., 2013). A rare butyrolactone lignan named arctiidilactone, and 11 novel butyrolactone lignans [arctigenin-4-O- $\beta$-D-gentiobioside, arctigenin-4-O- $\alpha-\mathrm{D}-$ galactopyranosyl- $(1 \rightarrow 6)-O-\beta$-D-glucopyranoside, arctigenin-4$O-\beta$-D-apiofuranosyl- $(1 \rightarrow 6)-O-\beta$-D-glucopyranoside, $\quad 5^{\prime}$ propanediolmatairesinoside, $\left(7^{\prime} \mathrm{R}, 8 \mathrm{R}, 8^{\prime} \mathrm{R}\right)$-rafanotrachelogenin4-O- $\beta$-D-glucopyranoside, $\quad\left(7^{\prime} \mathrm{S}, 8 \mathrm{R}, 8^{\prime} \mathrm{R}\right)$-rafanotrachelogenin-4$O$ - $\beta$-D-glucopyranoside, $\quad\left(7 \mathrm{~S}, 8 \mathrm{~S}, 8^{\prime} \mathrm{R}\right)-4,7$-dihydroxy-3,3', $\quad 4$ trimethoxyl-9-oxo benzylbutyrolactone lignan-4$O-\beta$-D-glucopyranoside, $\quad\left(7 \mathrm{R}, 8 \mathrm{~S}, 8^{\prime} \mathrm{R}\right) \quad-4,7,4^{\prime}$-trihydroxy-3,3'dimethoxyl-9-oxo dibenzylbutyrolactone lignan-4-O- $\beta-\mathrm{D}$ glucopyranoside, (7S,8S,8'R)-4,7-dihydroxy-3,3', ,4'-trimethoxyl9-oxo dibenzylbutyrolactone lignan, arctiidilactone, arctiiapolignan A and arctiisesquineolignan A] were determined in A. lappa fruits (Yang et al., 2015). Phylligenin, matairesinoside and pinoresinol were reported only in the fruits of A. lappa (Boldizsar et al., 2010). Also, 2 secolignans, styraxlignolide D and styraxlignolide E (Yang et al., 2015) and 4 new neolignan glucosides namely, (8R)-4,9,9'-trihydroxy-3,3'-dimethoxy-7oxo-8-O-4' -neolignan-4-O- $\beta$-D-glucopyranoside, $\quad(7 \mathrm{~S}, 8 \mathrm{R})-4$, $7,9,9^{\prime}$-tetrahydroxy-3,3' -dimethoxy-8-O-4' -neolignan- $9^{\prime}-O-\beta$-D apiofuranosyl- $(1 \rightarrow 6)-O-\beta$-D-glucopyranoside, $\left(7^{\prime} S, 8^{\prime} \mathrm{R}, 8 \mathrm{~S}\right)-4,4^{\prime}$, $9^{\prime}$-trihydroxy-3,3'-dimethoxy - -7',9-epoxylignan-7-oxo-4-O- $\beta$ D-glucopyranoside and (7R, 8S)-dihydrodehydrodiconiferyl alcohol-7'-oxo-4-O- $\beta$-D-glucopyranoside are reported from A. lappa fruits (Huang X.Y. et al., 2015). Besides, phytochemical analysis of $A$. lappa fruits revealed the existence of 2 more lignans named arctiisesquineolignan $\mathrm{B}$ and arctiiphenolglycoside $\mathrm{A}$ ( $\mathrm{He}$ et al., 2016). Bioassay-guided separation and purification of hydroethanolic extracts of A. lappa fruits allowed to identify a 


\section{Non-volatile constituents of Arctium species}
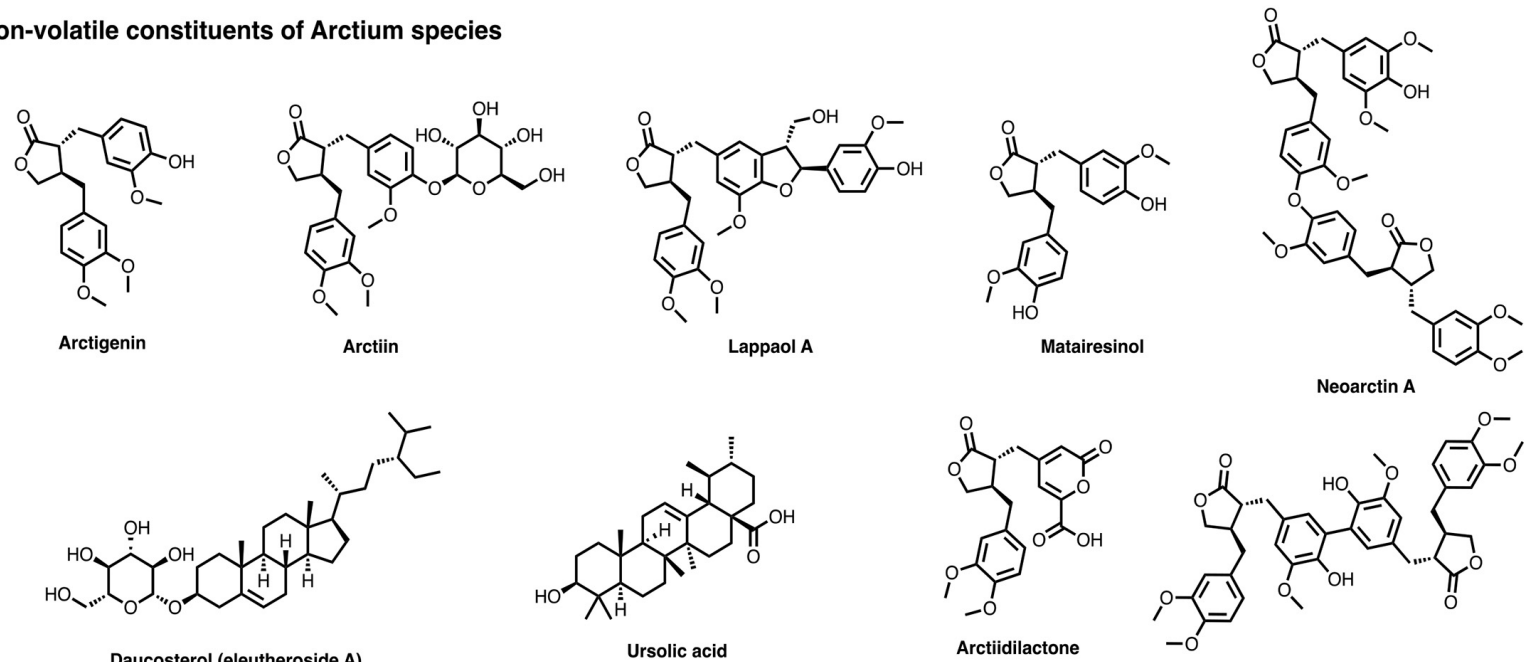

Daucosterol (eleutheroside A)

Ursolic acid

Diarctigenin

Volatile constituents of Arctium species

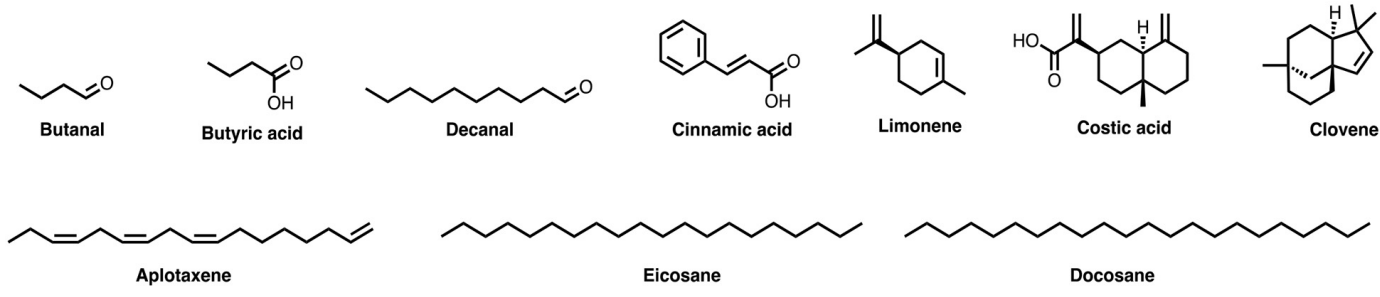

FIGURE 2 | Chemical structures of several relevant components present in Arctium species.

new lignan, $(+)$-7,8-didehydroarctigenin along with arctigenin and matairesinol identified previously (Matsumoto et al., 2006).

\section{Fatty Acids and Esters}

In search of $\alpha$-glucosidase inhibitory compounds, Miyazawa et al. (2005) found 11 compounds in A. lappa methanol extract. Among them, 10 compounds belonged to fatty acids. The identified compounds were linolenic acid, linoleic acid, methyl linoleate, methyl oleate, methyl linolenate, oleic acid, palmitic acid, methyl palmitate, methyl stearate, and stearic acid. Methanol extract from Arctium lappa L. which was found to contain sitosterol- $\beta$-D-glucopyranoside, methyl palmitate, methyl linoleate and methyl linoleneate showed an inhibitory activity against $\alpha$-glucosidase at 97.3, $73.4,66.5$, and $68.5 \%$ respectively at a concentration of 200.0 MM (Miyazawa et al., 2005). Later, Kuo et al. (2012) identified methyl methyl $\alpha$-linolenate, linolenic acid and methyl oleate as the chief constituents in the $n$-hexane fraction of $A$. lappa root (Kuo et al., 2012). The presence of linoleic acid, oleic acid, palmitic acid and stearic acid were also reported from A. lappa fruits (Boldizsar et al., 2010). Fatty acid composition of $A$. tomentosum seeds showed the occurrence of docosanoic acid, hexadecanoic acid, 9hexadecenoic acid, 9,12-octadecadienoic acid, oxiraneoctanoic acid, eicosanoic acid, cis-13-eicosenoic acid, and tetracosanoic acid (Zhou et al., 2011).

\section{Acetylenic Compounds}

From the roots of A. lappa, Washino et al. (1986) isolated and characterized 9 sulfur-containing acetylenic compounds namely, arctinone-a, arctinone-b, arctinol-a, arctinol-b, arctinal, arctic acid-b, arctic acid-c, methyl arctate-b, and arctinone-a acetate. Based on the chemical and spectral analysis, it was found that all these compounds were derivatives of $5^{\prime}$ - (1-propynyl)2,2'-bithienyl-5-yl. Later, the occurrence of few guaianolides linked with a sulfur-containing acetylenic compounds namely dehydrodihydrocostus lactone, dehydrocostus lactone, lappaphen-a and lappaphen-b were discovered from the acetone extracts of A. lappa roots (Washino et al., 1986, 1987). Several bioactivities of the key A. lappa constituents have been well-described in literature including antibacterial and antifungal activities of acetylenic compounds (Takasugi et al., 1987) and anti-edematogenic activity on carrageenan-induced paw edema (Carlotto et al., 2016).

\section{Phytosterols}

Daucosterol, a natural phytosterol-like compound, was obtained from the seeds of A. lappa (Ahangarpour et al., 2017). The fruits of $A$. tomentosum are reported to contain 2 steroids, such as daucosterol and $\beta$-sitosterol. Using bioactivity-guided fractionation, daucosterol and $\beta$-sitosterol were recovered from the ethanolic extract (95\%) of A. lappa seeds (Ming et al., 2004). Later, sitosterol-beta-D-glucopyranoside was found in the 
methanolic extracts of A. lappa (Miyazawa et al., 2005). Also, daucosterol and $\beta$-sitosterol compounds were detected from the chloroform extracts of A. lappa roots (Han et al., 2013). It was shown that phytosterol daucosterol inhibited cancer cell proliferation by inducing autophagy through reactive oxygen species-dependent manner (Zhao et al., 2015), and exhibited immunoregulatory activity by inducing protective Th1 immune response (Lee et al., 2007).

\section{Polysaccharides}

For the first time, Fuchigami et al. (1990; Ferracane et al., 2010) determined the pectic polysaccharides in edible A. lappa roots. Later investigations revealed the occurrence of several kinds of polysaccharides such as pectic substance, rhamnogalacturonan with neutral sugars, hemicellulose (arabinan, arabinogalactan, galactan, xylan, and xyloglucan), galacturonic acid, glucose, galactose, arabinose, rhamnose, mannose, and cellulose in cell walls of A. lappa and A. minus roots and leaves (Kato and Watanabe, 1993; Carlotto et al., 2016). Also, arabinose, glucose, galactose, rhamnose, and raffinose are reported from fruits of A. lappa (Boldizsar et al., 2010). The xyloglucan characterized from A. minus comprised a repeated unit of oligosaccharides of hepta- $($ Glc-Xyl $=4: 3)$, deca- $($ Glc-Xyl-Gal-Fuc $=4: 3: 2: 1)$ and nona-(Glc-Xyl-Gal-Fuc $=4: 3: 1: 1)$ saccharides in the ratio of 14:5:12 (Kato and Watanabe, 1993). Biologically active inulintype fructofuranans and other fructooligosaccharides have been identified from the roots of $A$. lappa (Kardosova et al., 2003). Inulin, a fiber comprising oligomers and polymers of fructose units linked by $\beta(2 \rightarrow 1)$ fructosyl-fructose bonds, has also been reported in the roots of A. lappa (Rajasekharan et al., 2015). A water-soluble polysaccharide fructan with a molecular weight of 4,600 Da, named as ALP1, was purified from A. lappa root and was composed of fructose and glucose in the molar ratio of 13:1. They were linked in $\rightarrow(1)$-Fruf-(2) $\rightarrow$, Fruf-(2) $\rightarrow$ and Glcp-(1) $\rightarrow$ (Liu et al., 2014). The structure was similar to the crude fructan obtained previously by Kardosova et al. (2003). In A. tomentosum, the glucofructans content is $24 \%$, constituted by a polymer of 2 inulin type (GF-A and GF-B) and 1 graminan (a mixed type of glucofructans containing 1,2- and 2-6 bonds) type polysaccharides. HPTLC method was developed by Olennikov and Tankhaeva (2011) to quantify fructans in A. tomentosum and A. lappa (Olennikov and Tankhaeva, 2011). Two sugar alcohols, mannitol and sorbitol were reported from the fruits of A. lappa (Boldizsar et al., 2010). The yield of inulin from A. lappa root was successfully increased by adopting an ultrasonic extraction technology (Milani et al., 2011). It was indicated that water-soluble polysaccharide from A. lappa could significantly ameliorate the dysregulation of pro-inflammatory cytokines (IL$1 \beta$, IL- 6 , and TNF- $\alpha$ ) and anti-inflammatory cytokine (IL-10) caused by colitis (Wang et al., 2019).

\section{Caffeoylquinic Acid Derivatives (Carboxylic Acids)}

Caffeoylquinic acids are the major bioactive phenolic compounds of Arctium species and impart superior antioxidant properties to the plant. The roots of A. lappa were reported to contain caffeoylquinic acid derivatives such as 1-0-,5-O-dicaffeoylquinic acid, 1-0-,5-O-dicaffeoyl-3-Osuccinylquinic acid, 1-0,-5-O-dicaffeoyl-4-O-succinylquinic acid, 1-0-,5-O-dicaffeoyl-3-O-,4-O-disuccinylquic acid and 1-0-,3-0,5-O-tricaffeoyl-4-O- succinylquinic acid (Maruta et al., 1995). Chlorogenic acid content is much higher than the caffeic acid and both occur mainly in the skin of A. lappa roots (Chen et al., 2004). HPTLC analysis was used as a chemical profiling tool to estimate chlorogenic acid in A. lappa roots. The content ranged from 0.107 to $0.140 \%$. Lin and Harnly (2008) and Liu et al. (2012) identified several compounds, including 5-sinapoylquinic acid, 3-sinapoyl-5-caffeoylquinic acid, 3-sinapoyl-5-caffeoyl-1- methoxyoxaloylquinic acid, 4-sinapoyl-5-caffeoyl-1-methoxyoxaloylquinic acid, 1,4-dicaffeoylquinic acid, 3,4-dicaffeoylquinic acid, 4,5-dicaffeoylquinic acid, 3,5-dicaffeoylquinic acid, 3,5-dicaffeoyl-1-methoxyoxaloylquinic acid, 3-feruloyl- 5caffeoylquinic acid, 4,5-dicaffeoyl-1-methoxyoxaloylquinic acid, 3,5-dicaffeoyl-1- methoxyoxaloylquinic acid, 3-feruloyl-5caffeoylquinic acid, 4,5-dicaffeoyl-1- methoxyoxaloylquinic acid, 3,4,5-tricaffeoylquinic acid, 1,4,5-tricaffeoylquinic acid and 1,4,5-tricaffeoyl-3-methoxyoxaloylquinic acid from the roots of A. lappa. Jaric et al. (2007) and Jaiswal and Kuhnert (2011) have characterized succinic, fumaric and malic acid-containing chlorogenic acid from the roots of $A$. lappa. These compounds included 3succinoyl-4,5-dicaffeoyl, 1,5-dicaffeoyl-4-succinoylquinic acid, 1,5-dicaffeoyl-3-succinoylquinic acid, 3,4-dicaffeoyl5-succinoylquinic acid, 1,5-dicaffeoyl-4-fumaroylquinic acid, 1,3-dicaffeoyl-5-fumaroylquinic acid, 1,4-dicaffeoyl3-maloylquinic acid, 1,5-dicaffeoyl-3-maloylquinic acid and 1,5-dicaffeoyl-4-maloylquinic acid, 1,3,5-tricaffeoyl-4succinoylquinic acid, 1,5-dicaffeoyl-3,4-disuccinoylquinic acid, 1,5-dicaffeoyl-3-fumaroyl-4-succinoylquinic acid, 1-fumaroyl3,5-dicaffeoyl-4-succinoylquinic acid, 1,5-dicaffeoyl-3-succinoyl4-dimaloylquinic acid and dicaffeoyldimaloylquinic acid. Further, Liu et al. (2012) isolated and identified 12 caffeoylquinic acids in both seeds and roots of $A$. lappa. The identified compounds included chlorogenic acid, 1,5-di-O-caffeoylquinic acid, 1,3-di-O-caffeoylquinic acid, dicaffeoyl-maloylquinic acid, dicaffeoyl-maloylquinic acid, 1,3-di-O-caffeoylquinic acid, 1,5-di-O-caffeoyl-3-O-maloylquinic acid, 1,5-di-O-caffeoyl-3$O$-succinoylquinic acid, 1,5-di-O-caffeoyl-4-O-maloylquinic acid, dicaffeoyl-dimaloylquinic acid, 1,5-di-O-caffeoylquinic acid, 1,5-di-O-caffeoyl-3-O-succinoyl-4-Omaloyquinic acid, 1,5-di-O-caffeoyl-3,4-di-O-succinoylquinic acid, and 1,3,5-tri-Ocaffeoyl-4-O-succinoylquinic acid. In addition, phytochemical analysis of root extracts of A. lappa showed the occurrence of 8 additional isomers of hydroxycinnamic acids (Liu et al., 2012; Tousch et al., 2014). An average content of chlorogenic acid, 1-O-5-O-dicaffeoylquinic acid and 1,5-dicaffeoyl- 3succinylquinic acid was observed to be between 1.7 and $7.9 \mathrm{mg} / \mathrm{g}$ dry weight of roots (Wang et al., 2001). Two new neolignan glucosides named $(70 \mathrm{~S}, 80 \mathrm{R}, 8 \mathrm{~S})-4,40,90-$ trihydroxy-3,30-dimethoxy-70,9-epoxylignan-7-oxo-4-O-b-Dglucopyranosyl-40-O-b-D-glucopyranoside and (7S, 8R)4,7,9,90-tetrahydroxy-3,30-dimethoxyl- 70-oxo-8-40oxyneolignan-4-O-b-D-glucopyranoside were determined from 
the fruit extract of A. lappa (Yang et al., 2012). The occurrence of phenolic acids, caffeic acid, cynarin and chlorogenic acid has been reported for the first time in A. lappa seeds and leaves (Ferracane et al., 2010; Tang et al., 2014; Lou et al., 2016) and later in both seeds and roots (Predes et al., 2011). Chlorogenic acid was determined by Tardio et al. (2005) in the seeds of A. lappa. Further, UPLC analysis revealed the presence of caffeic acid, benzoic acid and $p$-coumaric acid in the leaves of $A$. lappa (Tardio et al., 2005; Lou et al., 2010a,b). From the ethyl acetate and $n$-butanol fractions of $A$. lappa, 1,5-O-two caffeoylquinic acids, succinic acid and 5-hydroxy maltol were identified for the first time (Han et al., 2013). Likewise, HPLC and UPLC with photodiode array (PDA) detector were used to quantify caffeoyl esters, chlorogenic acid and 1,5-dicaffeoylquinic acid in aerial parts and root samples of A. lappa (Haghi et al., 2013). Two more phenolic compounds, namely, coumaroylquinic acid and caffeoyl-hexose-hydroxyphenol were identified by Rajasekharan et al. (2015) in the root extracts of A. lappa (Rajasekharan et al., 2015). It was reported that caffeoylquinic acids and their derivatives show multiple pharmacological activities including decrease in diet-induced obesity via modulation of PPAR $\alpha$ and LXR $\alpha$ transcription (Huang K. et al., 2015) and anti-ulcerogenic effect (Lee et al., 2010).

\section{Flavonoids}

The reported flavonoids include flavonols, flavones, and their glycosides. Two major constituents, namely rutin and isoquercetin, along with few other minor flavonoids including kaempferol-3-O-rhamnoglucoside, quercimeritrin and astragalin were identified in the ethanolic extracts of $A$. minus leaves (Saleh and Bohm, 1971). Likewise, the occurrence of quercetin3-O-rhamnoside was reported from the leaves of A. lappa. Later, the presence of phenolic compounds such as quercetin, quercitrin, rutin, and luteolin have been reported in seeds, fruits, leaves and roots of A. lappa (Saleh and Bohm, 1971; Tamayo et al., 2000; Yu et al., 2003; Lou et al., 2010a,b, 2016; Predes et al., 2011; Liu et al., 2012; Tang et al., 2014). Also, few isoflavone derivatives including genistein, nobilein, biachanin $\mathrm{A}$ and tangeretin have been detected in A. lappa roots (Eberding et al., 2007). A comparative study has shown the existence of chemical differences within the A. lappa organs (Ferracane et al., 2010). According to them, luteolin and quercetin rhamnoside were detected in roots whereas rutin, quercetin, quercitrin and luteolin in leaves. On the other hand, no flavonoids were found in the seeds of $A$. lappa. Two more flavonols, namely quercetin 3-O-glucuronide and quercetin 3-vicianoside, were identified by Rajasekharan et al. (2015) in the root extracts of A. lappa.

\section{Terpenoids}

The fruits of $A$. lappa were found to contain $\beta$-eudesmol, a sesquiterpene alcohol (Rajasekharan et al., 2015; Yang et al., 2015). Pentacyclic triterpenoids, such as ursolic and oleanolic acids were detected by Han et al. in the ethanolic extract of A. lappa roots (Han et al., 2013). Arctiopicrin and onopordopicrin are the sesquiterpene lactones isolated from the leaf extract of A. lappa (Barbosa et al., 1993; Machado et al., 2012). Arctiopicrin occurrence is also evidenced in A. lappa. Later, few more sesquiterpene lactones, namely dehydromelitensin-8(4'-hydroxymethacrylate), dehydromelitensin, and melitensin and a norisoprenoid along with 2 more terpenes such as dehydrovomifoliol and loliolide were identified in A. lappa leaf (Machado et al., 2012). Onopordopicrin, a germacranolide sesquiterpene lactone was isolated from the aerial parts of A. nemorosum (Zimmermann et al., 2012). Two triterpenoids, namely $3 \alpha$-acetoxy-hop-22(29)-ene and $3 \alpha$-hydroxylanosta5,15 -diene were isolated from the leaves of $A$. lappa (Jaiswal and Kuhnert, 2011).

\section{Others}

From the concentrated sap obtained from A. lappa roots (A. lappa and $A$. tomentosum), $\beta$-asparagine was isolated for the first time (Boldizsar et al., 2010). The carotenoid crocin was reported to occur in the leaves of A. lappa (Lou et al., 2016).

\section{Volatile Compounds}

A total of 101 volatile chemical constituents were identified in A. lappa. The details of these compounds are partially summarized in Table 2 and described in this section. Carboxylic acids and fatty acids were more prevalent in A. lappa. On the other hand, there are no available literatures on the identification of volatile components in other Arctium species. The chemical structures of some compounds from Arctium species are shown in Figure 2.

\section{Hydrocarbons}

Fourteen hydrocarbon compounds, aplotaxene, clovene, dihydroaplotaxene, eicosane, 1-heptadecene, heptacosane, hexacosane, nonadecane, 2-naphthalenemethanol, 1pentadecene, pentacosane, pentadecane, tetracosane, and tetradecane were detected from the roots, seeds, and leaves of A. lappa (Washino et al., 1986; Wang et al., 2004). In addition, docosane, eicosane, heptacosane, hexacosane, tetracosane, and pentadecane were found only in roots and leaves. Docosane was found only in leaves, while seeds of A. lappa contained only eicosane.

\section{Aldehydes}

Nineteen aldehydes, namely, benzaldehyde, butanal, decanal, dodecanal, heptanal, hexanal, (Z)-3-hexenal, (E)-2-hexenal, 2-methylpropanal, 3-methylbutanal, nonanal, octanal, (E)-2octanal, phenylacetaldehyde, pentanal, propanal, tridecanal, 4methoxybenzaldehyde, and undecanal were found as root volatile compounds in A. lappa (Washino et al., 1986, 1987; Wang et al., 2004). Interestingly, only the alkyl aldehyde nonanal was present in all plant parts such as roots, leaves, and seeds (Washino et al., 1986, 1987).

\section{Methoxypyrazines}

Seven methoxypyrazines, such as 2-methoxy-3methylpyrazine, 2-methoxy-3- propylpyrazine, 2-isopropyl3-methyoxylpyrazine, 2-sec-butyl-3-methoxypyrazine, 2butyl-3- methoxypyrazine, 2-isobutyl-3-methoxypyrazine, and 2-isoamyl-3-methoxypyrazine were detected in roots of $A$. lappa (Washino et al., 1986, 1987). 
TABLE 2 | Volatile constituents of Arctium spp.

\begin{tabular}{|c|c|c|c|c|c|c|}
\hline S. No & Compound name & Formula & Species & Plant origin/part & Analytical method & References \\
\hline 1 & Aplotaxene & $\mathrm{C}_{17} \mathrm{H}_{28}$ & A. lappa & Roots & GCMS & Washino et al., 1986, 1987 \\
\hline 3 & Dihydroaplotaxene & $\mathrm{C}_{17} \mathrm{H}_{30}$ & A. lappa & Roots & GCMS & Washino et al., 1986, 1987 \\
\hline 4 & Docosane & $\mathrm{C}_{22} \mathrm{H}_{46}$ & A. lappa & Leaves & GCMS & Aboutabl et al., 2013 \\
\hline 5 & Eicosane & $\mathrm{C}_{20} \mathrm{H}_{42}$ & A. lappa & Roots/leaves/seeds & GCMS & Aboutabl et al., 2013 \\
\hline 7 & Heptacosane & $\mathrm{C}_{27} \mathrm{H}_{56}$ & A. lappa & Roots/leaves & GCMS & Aboutabl et al., 2013 \\
\hline 8 & Hexacosane & $\mathrm{C}_{26} \mathrm{H}_{54}$ & A. lappa & Roots/leaves & GCMS & Aboutabl et al., 2013 \\
\hline 9 & Nonadecane & $\mathrm{C}_{19} \mathrm{H}_{40}$ & A. lappa & Leaves & GCMS & Aboutabl et al., 2013 \\
\hline 10 & 2-Naphthalenemethanol & $\mathrm{C}_{11} \mathrm{H}_{10} \mathrm{O}$ & A. lappa & Roots & GCMS & Wang et al., 2004 \\
\hline 11 & 1-Pentadecene & $\mathrm{C}_{15} \mathrm{H}_{30}$ & A. lappa & Roots & GCMS & Washino et al., 1986, 1987 \\
\hline 12 & Pentacosane & $\mathrm{C}_{25} \mathrm{H}_{52}$ & A. lappa & Roots & GCMS & Aboutabl et al., 2013 \\
\hline 15 & Aldehydes & & & & & \\
\hline 16 & Benzaldehyde & $\mathrm{C}_{7} \mathrm{H}_{6} \mathrm{O}$ & A. lappa & Roots & GCMS & $\begin{array}{l}\text { Washino et al., 1986, 1987; } \\
\text { Wang et al., } 2004\end{array}$ \\
\hline 17 & Butanal & $\mathrm{C}_{4} \mathrm{H}_{8} \mathrm{O}$ & A. lappa & Roots & GCMS & Washino et al., 1986, 1987 \\
\hline 18 & Decanal & $\mathrm{C}_{10} \mathrm{H}_{20} \mathrm{O}$ & A. lappa & Roots & GCMS & Washino et al., 1986, 1987 \\
\hline 19 & Dodecanal & $\mathrm{C}_{12} \mathrm{H}_{24} \mathrm{O}$ & A. lappa & Roots & GCMS & Washino et al., 1986, 1987 \\
\hline 20 & Heptanal & $\mathrm{C}_{7} \mathrm{H}_{14} \mathrm{O}$ & A. lappa & Roots & GCMS & Washino et al., 1986, 1987 \\
\hline 21 & Hexanal & $\mathrm{C}_{6} \mathrm{H}_{12} \mathrm{O}$ & A. lappa & Roots & GCMS & Washino et al., 1986, 1987 \\
\hline 22 & (Z)-3-Hexenal & $\mathrm{C}_{6} \mathrm{H}_{10} \mathrm{O}$ & A. lappa & Roots & GCMS & Washino et al., 1986, 1987 \\
\hline 23 & (E)-2-Hexenal & $\mathrm{C}_{6} \mathrm{H}_{10} \mathrm{O}$ & A. lappa & Roots & GCMS & Washino et al., 1986, 1987 \\
\hline 24 & 2-Methylpropanal & $\mathrm{C}_{4} \mathrm{H}_{8} \mathrm{O}$ & A. lappa & Roots & GCMS & Washino et al., 1986, 1987 \\
\hline 32 & Tridecanal & $\mathrm{C}_{13} \mathrm{H}_{26} \mathrm{O}$ & A. lappa & Roots & GCMS & Washino et al., 1986, 1987 \\
\hline 33 & 4-Methoxybenzaldehyde & $\mathrm{C}_{8} \mathrm{H}_{8} \mathrm{O}_{2}$ & A. lappa & Roots & GCMS & Washino et al., 1986, 1987 \\
\hline \multirow[t]{2}{*}{34} & Undecanal & $\mathrm{C}_{11} \mathrm{H}_{22} \mathrm{O}$ & A. lappa & Roots & & Washino et al., 1986, 1987 \\
\hline & Methoxypyrazines & & & & & \\
\hline 35 & 2-Methoxy-3-methylpyrazine & $\mathrm{C}_{6} \mathrm{H}_{8} \mathrm{~N}_{2} \mathrm{O}$ & A. lappa & Roots & GCMS & Washino et al., 1986, 1987 \\
\hline 36 & 2-Isopropyl- 3-methyoxylpyrazine & $\mathrm{C}_{8} \mathrm{H}_{12} \mathrm{~N}_{2} \mathrm{O}$ & A. lappa & Roots & GCMS & Washino et al., 1986, 1987 \\
\hline 37 & 2-Methoxy-3- propylpyrazine & $\mathrm{C}_{8} \mathrm{H}_{12} \mathrm{~N}_{2} \mathrm{O}$ & A. lappa & Roots & GCMS & Washino et al., 1986, 1987 \\
\hline 38 & 2-sec-Butyl-3-methoxypyrazine & $\mathrm{C}_{9} \mathrm{H}_{14} \mathrm{~N}_{2}$ & A. lappa & Roots & GCMS & Washino et al., 1986, 1987 \\
\hline 39 & 2-Isobutyl-3-methoxypyrazine & $\mathrm{C}_{9} \mathrm{H}_{14} \mathrm{~N}_{2} \mathrm{O}$ & A. lappa & Roots & GCMS & Washino et al., 1986, 1987 \\
\hline 40 & 2-Butyl-3- methoxypyrazine & $\mathrm{C}_{9} \mathrm{H}_{14} \mathrm{~N}_{2}$ & A. lappa & Roots & GCMS & Washino et al., 1986, 1987 \\
\hline \multirow[t]{2}{*}{41} & 2-Isoamyl-3-methoxypyrazine & $\mathrm{C}_{9} \mathrm{H}_{14} \mathrm{~N}_{2} \mathrm{O}$ & A. lappa & Roots & GCMS & Washino et al., 1986, 1987 \\
\hline & Fatty acids/Carboxylic acids & & & & & \\
\hline 42 & Acetic acid & $\mathrm{CH}_{3} \mathrm{COOH}$ & A. lappa & Roots & GCMS & Washino et al., 1986, 1987 \\
\hline 43 & Benzoic acid & $\mathrm{C}_{7} \mathrm{H}_{6} \mathrm{O}_{2}$ & A. lappa & Roots & GCMS & Washino et al., 1986, 1987 \\
\hline 44 & Butyric acid & $\mathrm{C}_{4} \mathrm{H}_{8} \mathrm{O}_{2}$ & A. lappa & Roots & GCMS & Washino et al., 1986, 1987 \\
\hline 45 & Cinnamic acid & $\mathrm{C}_{9} \mathrm{H}_{8} \mathrm{O}_{2}$ & A. lappa & Roots & GCMS & Washino et al., 1986, 1987 \\
\hline 46 & Costic acid & $\mathrm{C}_{15} \mathrm{H}_{22} \mathrm{O}_{2}$ & A. lappa & Roots & GCMS & Washino et al., 1986, 1987 \\
\hline 47 & Decanoic acid & $\mathrm{C}_{10} \mathrm{H}_{20} \mathrm{O}_{2}$ & A. lappa & Roots & GCMS & Washino et al., 1986, 1987 \\
\hline 48 & Dodecanoic acid & $\mathrm{C}_{12} \mathrm{H}_{24} \mathrm{O}_{2}$ & A. lappa & Roots & GCMS & Washino et al., 1986, 1987 \\
\hline
\end{tabular}


TABLE 2 | Continued

\begin{tabular}{|c|c|c|c|c|c|c|}
\hline S. No & Compound name & Formula & Species & Plant origin/part & Analytical method & References \\
\hline 50 & Hexanoic acid & $\mathrm{C}_{3} \mathrm{H}_{6} \mathrm{O}_{2}$ & A. lappa & Roots & GCMS & Washino et al., 1986, 1987 \\
\hline 52 & (E)-3-Hexenoic acid & $\mathrm{C}_{6} \mathrm{H}_{10} \mathrm{O}_{2}$ & A. lappa & Roots & GCMS & Washino et al., 1986, 1987 \\
\hline 53 & Heptanoic acid & $\mathrm{C}_{7} \mathrm{H}_{14} \mathrm{O}_{2}$ & A. lappa & Roots & GCMS & Washino et al., 1986, 1987 \\
\hline 55 & Linoleic acid & $\mathrm{C}_{18} \mathrm{H}_{32} \mathrm{O}_{2}$ & A. lappa & Roots & GCMS & Wang et al., 2004 \\
\hline 56 & 2, 3-Hydroxyoctanoic acid & $\mathrm{C}_{8} \mathrm{H}_{16} \mathrm{O}_{3}$ & A. lappa & Roots & GCMS & Washino et al., 1986, 1987 \\
\hline 57 & 2-Methylpropionic acid & $\mathrm{C}_{4} \mathrm{H}_{8} \mathrm{O}_{2}$ & A. lappa & Roots & GCMS & Washino et al., 1986, 1987 \\
\hline 58 & 2-Methylbutyric acid & $\mathrm{C}_{5} \mathrm{H}_{10} \mathrm{O}_{2}$ & A. lappa & Roots & GCMS & Washino et al., 1986, 1987 \\
\hline 59 & 3-Methoxybenzoic acid & $\mathrm{C}_{8} \mathrm{H}_{8} \mathrm{O}_{3}$ & A. lappa & Roots & GCMS & Washino et al., 1986, 1987 \\
\hline 60 & Methyl palmitate & $\mathrm{C}_{17} \mathrm{H}_{34} \mathrm{O}_{2}$ & A. lappa & Roots/seeds & GCMS & $\begin{array}{l}\text { Washino et al., 1986, 1987; } \\
\text { Aboutabl et al., } 2013\end{array}$ \\
\hline 64 & Nonanedioic acid & $\mathrm{C}_{9} \mathrm{H}_{16} \mathrm{O}_{4}$ & A. lappa & Roots & GCMS & Washino et al., 1986;, 1987 \\
\hline 65 & (E)-3-nonenoic acid & $\mathrm{C}_{9} \mathrm{H}_{16} \mathrm{O}_{2}$ & A. lappa & Roots & GCMS & Washino et al., 1986, 1987 \\
\hline 66 & Octanoic acid & $\mathrm{C}_{8} \mathrm{H}_{16} \mathrm{O}_{2}$ & A. lappa & Roots & GCMS & Washino et al., 1986, 1987 \\
\hline 67 & (E)-3-Octenoic acid & $\mathrm{C}_{8} \mathrm{H}_{14} \mathrm{O}_{2}$ & A. lappa & Roots & GCMS & Washino et al., 1986, 1987 \\
\hline 68 & Octadecanoic acid & $\mathrm{C}_{18} \mathrm{H}_{36} \mathrm{O}_{2}$ & A. lappa & Roots & GCMS & Washino et al., 1986, 1987 \\
\hline 69 & Octadecanoic acid methyl ester & $\mathrm{C}_{18} \mathrm{H}_{36} \mathrm{O}_{2}$ & A. lappa & Seeds & GCMS & Aboutabl et al., 2013 \\
\hline 70 & Pentanoic acid & $\mathrm{C}_{5} \mathrm{H}_{10} \mathrm{O}_{2}$ & A. lappa & Roots & GCMS & Washino et al., 1986, 1987 \\
\hline 71 & Phenylacetic acid & $\mathrm{C}_{8} \mathrm{H}_{8} \mathrm{O}_{2}$ & A. lappa & Roots & GCMS & Washino et al., 1986, 1987 \\
\hline 72 & Phenylpropionic acid & $\mathrm{C}_{9} \mathrm{H}_{10} \mathrm{O}_{2}$ & A. lappa & Roots & GCMS & Washino et al., 1986, 1987 \\
\hline 73 & Propionic acid & $\mathrm{C}_{3} \mathrm{H}_{6} \mathrm{O}_{2}$ & A. lappa & Roots & GCMS & Washino et al., 1986, 1987 \\
\hline 79 & Carvomenthone & $\mathrm{C}_{10} \mathrm{H}_{18} \mathrm{O}$ & A. lappa & Roots/leaves & GCMS & Aboutabl et al., 2013 \\
\hline 80 & Geraniol & $\mathrm{C}_{10} \mathrm{H}_{18} \mathrm{O}$ & A. lappa & Seeds & GCMS & Aboutabl et al., 2013 \\
\hline 81 & Linalool & $\mathrm{C}_{10} \mathrm{H}_{18} \mathrm{O}$ & A. lappa & Seeds & GCMS & Aboutabl et al., 2013 \\
\hline 82 & Thymol & $\mathrm{C}_{10} \mathrm{H}_{14} \mathrm{O}$ & A. lappa & Seeds & GCMS & Aboutabl et al., 2013 \\
\hline 83 & Z-citral & $\mathrm{C}_{10} \mathrm{H}_{16} \mathrm{O}$ & A. lappa & Seeds & GCMS & Aboutabl et al., 2013 \\
\hline \multirow[t]{2}{*}{84} & E-citral & $\mathrm{C}_{10} \mathrm{H}_{16} \mathrm{O}$ & A. lappa & Seeds & GCMS & Aboutabl et al., 2013 \\
\hline & Sesquiterpenoids & & & & & \\
\hline 85 & Dehydrocostus lactone & $\mathrm{C}_{15} \mathrm{H}_{18} \mathrm{O}_{2}$ & A. lappa & Roots & GCMS & Washino et al., 1986, 1987 \\
\hline \multirow[t]{2}{*}{86} & Dehydrodihydrocostus lactone & $\mathrm{C}_{15} \mathrm{H}_{29} \mathrm{O}_{2}$ & A. lappa & Roots & GCMS & Washino et al., 1986, 1987 \\
\hline & Oxygenated sesquiterpenes & & & & & \\
\hline 87 & Caryophyllene oxide & $\mathrm{C}_{15} \mathrm{H}_{24} \mathrm{O}$ & A. lappa & Roots/leaves & GCMS & Aboutabl et al., 2013 \\
\hline \multirow[t]{2}{*}{88} & $\beta$-Costol & $\mathrm{C}_{15} \mathrm{H}_{24} \mathrm{O}$ & A. lappa & Roots & GCMS & Aboutabl et al., 2013 \\
\hline & Sesquiterpene Hydrocarbons & & & & & \\
\hline 89 & Aromadendrene & $\mathrm{C}_{15} \mathrm{H}_{24}$ & - & Roots/seeds & GCMS & Aboutabl et al., 2013 \\
\hline 90 & Caryophyllene & $\mathrm{C}_{15} \mathrm{H}_{24}$ & A. lappa & Roots & GCMS & Washino et al., 1986, 1987 \\
\hline 91 & $\gamma$-Cadinene & $\mathrm{C}_{15} \mathrm{H}_{24}$ & - & Roots/leaves/seeds & GCMS & Aboutabl et al., 2013 \\
\hline 92 & Cyperene & $\mathrm{C}_{15} \mathrm{H}_{24}$ & A. lappa & Roots & GCMS & Washino et al., 1986, 1987 \\
\hline 93 & $\beta$-Elemene & $\mathrm{C}_{15} \mathrm{H}_{24}$ & A. lappa & Roots & GCMS & $\begin{array}{l}\text { Washino et al., 1986, 1987; } \\
\text { Aboutabl et al., } 2013\end{array}$ \\
\hline
\end{tabular}


TABLE 2 | Continued

\begin{tabular}{|c|c|c|c|c|c|c|}
\hline S. No & Compound name & Formula & Species & Plant origin/part & Analytical method & References \\
\hline 94 & trans- $\beta$-Farnesene & $\mathrm{C}_{15} \mathrm{H}_{24}$ & A. lappa & Roots/leaves & GCMS & Aboutabl et al., 2013 \\
\hline 95 & $\alpha$-Guaiene & $\mathrm{C}_{15} \mathrm{H}_{24}$ & A. lappa & Roots & GCMS & Washino et al., 1986, 1987 \\
\hline 96 & Isoaromadendrene epoxide & $\mathrm{C}_{15} \mathrm{H}_{24} \mathrm{O}$ & - & Roots/leaves/seeds & GCMS & Aboutabl et al., 2013 \\
\hline 97 & Limonene & $\mathrm{C}_{10} \mathrm{H}_{16}$ & A. lappa & Leaves/seeds & GCMS & Washino et al., 1986, 1987 \\
\hline 98 & $\alpha$-Myrcene & $\mathrm{C}_{10} \mathrm{H}_{16}$ & A. lappa & Seeds & GCMS & Aboutabl et al., 2013 \\
\hline 99 & $\alpha$-Pinene & $\mathrm{C}_{10} \mathrm{H}_{16}$ & A. lappa & Roots/leaves & GCMS & Aboutabl et al., 2013 \\
\hline \multirow[t]{2}{*}{100} & Squalene & $\mathrm{C}_{30} \mathrm{H}_{50}$ & A. lappa & Seeds & GCMS & Aboutabl et al., 2013 \\
\hline & Sesquiterpene Alcohol & & & & & \\
\hline 101 & $\beta$-Copaen- $4 \alpha$-ol & $\mathrm{C}_{15} \mathrm{H}_{24} \mathrm{O}$ & - & Roots/leaves/seeds & GCMS & Aboutabl et al., 2013 \\
\hline
\end{tabular}

\section{Carboxylic Acids and Fatty Acids}

Twenty-two carboxylic acids namely acetic acid, benzoic acid, butyric acid, cinnamic acid, costic acid, dodecanoic acid, hexanoic acid, (E)-3-hexenoic acid, heptanoic acid, (E)-3-heptenoic acid, 2, 3-hydroxyoctanoic acid, 2methylpropionic acid, 2-methylbutyric acid, 3-methoxybenzoic acid, nonanoic acid, nonanedioic acid, pentanoic acid, phenylacetic acid, phenylpropionic acid, propionic acid, salicylic acid, and undecanoic acid were identified in A. lappa roots (Washino et al., 1986, 1987; Wang et al., 2004). Fatty acids such as decanoic acid, hexadecanoic acid, linoleic acid, octanoic acid, (E)-3-octenoic acid, octadecanoic acid, pentadecanoic acid, tridecanoic acid, and tetradecanoic acid were found in roots while ethyl oleate, methyl oleate, hexadecanoic acid, methyl palmitate, and octadecanoic acid methyl ester were identified in seeds of A. lappa (Washino et al., 1986, 1987; Wang et al., 2004).

\section{Monoterpenes and Sesquiterpenes}

Three alcoholic and one phenolic monoterpenoids (carvomenthone, geraniol, linalool, and thymol); 2 sesquiterpene lactones (dehydrocostus lactone and dehydrodihydrocostus lactone, isoaromadendrene epoxide); 2 oxygenated sesquiterpenes (caryophyllene oxide and $\beta$-costol) and 12 sesquiterpene hydrocarbons namely, aromadendrene, caryophyllene, $\gamma$-cadinene, cyperene, $\beta$-elemene, trans$\beta$-farnesene, $\alpha$-guaiene, limonene, myrcene, $\alpha$-pinene, and squalene were identified in A. lappa (Washino et al., 1986, 1987; Wang et al., 2004). Geraniol, linalool, thymol, aromadendrene, $\gamma$-cadinene, isoaromadendrene epoxide, limonene, $\alpha$-myrcene,

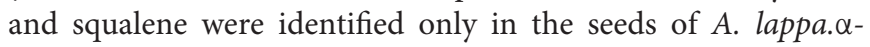
Pinene, isoaromadendrene epoxide, $\gamma$-cadinene, carvomenthone, and caryophyllene oxide were found in the roots and leaves.

\section{BIOACTIVITIES OF Arctium SPECIES}

Arctium lappa is widely used as an ethno-medicinal plant especially in North America, Asia and Europe, and is applied to treat various diseases including diabetes, gout, rheumatism, and skin problems (Chan et al., 2011; Azizov et al., 2012). A. lappa roots have been used as a vegetable in Japanese (referred to as 'gobo') and Korean cuisine. Its root has been used to treat constipation, mercury poisoning, upper respiratory infections, inflammation and oxidative stress in patients with knee osteoarthritis (MaghsoumiNorouzabad et al., 2016), while the leaves were efficacious in healing burns, rashes, and applied in women with labor condition (Force, 2001; Lewis and Elvin-Lewis, 2003; Amish Burn Study et al., 2014). A. lappa has also been found for the treatment of alopecia (loss of hair) among adults (Amish Burn Study et al., 2014). In Western countries, burdock is used as a remedy for several ailments ranging from arthritis, chronic inflammation, and various skin problems (e.g., scaly skin conditions such as psoriasis and eczema) to cancer treatment (Wu et al., 2010; Amish Burn Study et al., 2014).

Studies on the biological activities of extracts of different parts of $A$. lappa and compounds isolated thereof, were carried out and revealed antipyretic, antimicrobial, diuretic, diaphoretic, hypoglycaemic, antioxidant, anti-inflammatory, anti-hepatotoxicity, antiulcer, antimutagenicity, and antitumour activities.

\section{Anticancer Effects}

Arctium lappa fruit has been used in traditional medicine, and it is popular for its various anticancer effects. Arctigenin (ATG), a natural lignan product extracted from the seeds of Arctium lappa, has been shown to have estrogenic properties, that reduced the risk of osteoporosis, heart disease, and menopausal symptoms (Maxwell et al., 2017). It was found to possess antitumor effect by modulating the protein kinase activation pathway and hence rendering the tumor cells susceptible to effects of the nutrient-deprived environment (Awale et al., 2006). Later on, ATG was shown to induce apoptosis (programmed cell death) of estrogen receptor-negative cancer cells (MDA-MB-231) through the ROS/p38 MAPK pathway and epigenetic regulation of Bcl-2 by upregulating trimethylation of histone H3K9 (Hsieh et al., 2014). It was reported that ATG was able to inhibit cell proliferation and may induce apoptosis and cell cycle arrest at the G0/G1 phase in glioma cells (Maimaitili et al., 2017). In more detail, it was found that ATG increased the expression levels of p21, retinoblastoma and p53 proteins, and significantly decreased the expression levels of cyclin D1 and CDK4 proteins (Maimaitili et al., 2017). Furthermore, ATG was able to induce apoptosis in glioma cells, coupled with increased expression levels of cleaved caspase- 3 and the 
pro-apoptotic BCL2-associated X protein (Maimaitili et al., 2017). ATG-induced apoptosis was significantly suppressed by the pretreatment of cells with Z-DEVD-FMK, a caspase-3 inhibitor (Maimaitili et al., 2017). More recently, study by Lou et al. (2017) demonstrated ATG to significantly inhibit in vitro migration and invasion of human breast cancer cells (MDA-MB-231) by downregulation of MMP-2, MMP-9 and heparanase (Lou et al., 2017).

Extracts from A. lappa also showed selective antiproliferative activity against certain human cancer cell lines including K562, MCF-7 and 786-0 (Predes et al., 2011). Lappaol $\mathrm{F}$, a novel natural product isolated from the seeds of A. lappa, was found to suppress cancer cell growth in a dose-dependent manner in various human cancer cell lines through induction of G1 and G2 cell-cycle arrest. This effect was associated with strong induction of p21 and p27 and suppression of cyclin-dependent kinase 1 (CDK1) and cyclin B1 (Sun et al., 2014).

A. lappa is one of the herbs widely used by cancer patients in some Canadian populations to improve quality of life (QOL) and prevent cancer progression. A. lappa is one of the herbs constituting the two proprietary herbal products: Flor-Essence ${ }^{\circledR}$ and Essiac ${ }^{\circledR}$ suggested for prolong survival and the improvement of QOL among cancer patients (Tamayo et al., 2000).

\section{Antidiabetic Effects}

Root of A. lappa root has been found to mediate hypoglycemic activities making it a popular choice to be used as a traditional medicine in diabetes. Oral administration of burdock root ethanolic extract in streptozotocin-induced diabetic rats significantly lowered blood glucose and increased insulin level in the diabetic rats compared to the control diabetic group (Cao et al., 2012). Additionally, treatment with A. lappa extract also reduced the levels of serum total cholesterol (TC), triglycerides (TG) and low density lipoprotein (LDL), whereas high density lipoprotein (HDL) level was higher in the control rats. More recently in a similar study, Ahangarpour et al. (2017) investigated the antidiabetic and hypolipidemic properties of the root extract of A. lappa on nicotinamide-streptozotocin (NA-STZ)-induced type 2 diabetes in mice (Ahangarpour et al., 2017). The results show that root extract of $A$. lappa displays anti-diabetic effect at certain doses. It exerts its effects through hypolipidemic and insulinotropic properties and hence the root extract could serve successfully in treating patients with type 2 diabetes in the future. Moreover, sitosterol- $\beta$-D-glucopyranoside from burdock's root acts as a potent inhibitor of alpha-glucosidases, thereby having the potential to reduce glycogenolysis and help to decrease blood glucose level (Tousch et al., 2014). In addition, Zhao and Zhou (2015) demonstrated that trace elements (e.g., Na, K, Mn, Fe, and $\mathrm{Mg}$ ) present in the root and fruit extracts of $A$. lappa exhibit antidiabetic effects. While A. lappa constituents do reduce absorption of glucose, they also elevate inulin content in blood and slow digestion of carbohydrates to confer its anti-diabetic activities. The pharmacological mechanisms of A. lappa roots are slightly different from other classes of oral antihyperglycemic agents such as metformin. Metformin decreases hepatic glucose production, decreases intestinal absorption of glucose, and improves insulin sensitivity by increasing peripheral glucose uptake and utilization (Dumitrescu et al., 2015).

\section{Anti-oxidant, Hepatoprotective and Gastroprotective Activities}

It is believed that lignans and caffeoylquinic acids from A. lappa are of value because of their antioxidant capacity (Maruta et al., 1995; Mkrtchian et al., 1998; Jaiswal and Kuhnert, 2011) by which they can scavenge free radicals that are thought to play an important role in many diseases.

The hydroalcoholic extracts of burdock roots possess significant antioxidant potential as seen by the application of various assays. Very recently, Fierascu et al. (2018) quantified antioxidant potential of burdock extracts using DPPH (2,2diphenyl-1-picrylhydrazyl) and phosphomolybdate assays to demonstrate that burdock extracts have very high antioxidative activities, presumably due to the high content of polyphenols (Fierascu et al., 2018). The potent antioxidative property makes these extracts effective inhibitors of lipid peroxidation in rat liver homogenate in vitro (Duh, 1998) and an excellent hepatoprotective agent in vivo and in vitro (Lin et al., 2000). Due to its radical scavenging ability, A. lappa is also used to treat gastrointestinal ulcers (da Silva et al., 2013).

\section{Antimicrobial Effects}

Extracts of different parts of A. lappa have been investigated for their microbial-modulatory properties by many researchers. An organic extract from $A$. lappa has shown inhibiting properties toward the growth of Pseudomonas aeruginosa, Escherichia coli, Lactobacillus acidophilus, Streptococcus mutans, and Candida albicans residing in the teeth of the oral cavity (Gentil et al., 2006). Pereira et al. (2005) further reported potent growth inhibiting activities of A. lappa extract against a broad spectrum of oral microorganisms, specifically those associated with teeth infections, namely Enterococcus faecalis, Staphylococcus aureus, Pseudomonas aeruginosa, Bacillus subtilis, and Candida albicans (Pereira et al., 2005). Very recently, Fierascu et al. (2018) investigated antifungal potential of hydroalcoholic extract of burdock roots and observed that it is active against the fungal lines Aspergillus niger ATCC 15475 and Penicillium hirsutum ATCC 52323 (Fierascu et al., 2018). Fruit extract of A. lappa was tested (Dias et al., 2017) for in vitro antiviral properties against Herpes simplex virus type-1 (HSV-1) and was found to decrease viral load significantly at all concentrations tested $(400,50$, and $3.125 \mu \mathrm{g} / \mathrm{mL})$. At $400 \mu \mathrm{g} / \mathrm{mL}$ concentration, it showed comparable antiviral activity as acyclovir $(50 \mu \mathrm{g} / \mathrm{mL})$. Arctigenin, one of the key constituents of $A$. lappa extract, has shown potent activities against human immunodeficiency virus type-1 (HIV-1) both in vivo and in vitro presumably by increasing the expression of Heme oxygenase-2 (HO-2) and blocking HIV-1gag proteins (Schroder et al., 1990).

\section{Anti-inflammatory Effects}

Various parts of $A$. lappa demonstrated anti-inflammatory effects (Liu et al., 2005). Burdock extract is known to 
alleviate wound irritation and swelling and therefore has been traditionally used for healing burn wounds. This effect might be mediated through the inhibition of the cyclooxygenase-2 (COX-2) enzyme. Cyclooxygenase is a lipid metabolizing enzyme that catalyzes the oxygenation of polyunsaturated fatty acids. This process forms prostanoids, specifically eicosanoids, which are known to be potent cell signaling molecules connected to inflammatory processes (Charlier and Michaux, 2003). Phenolic compounds present in burdock extract (e.g., arctigenin, lappaol F, diarctigenin) are inhibitors of this enzyme (Zhao et al., 2009; Lee and Kim, 2010), thereby suppressing lipopolysaccharide (LPS)-stimulated NO production (Park et al., 2007) and pro-inflammatory cytokines secretion (including TNF- $\alpha$ and IL-6) in a dose-dependent manner (Zhao et al., 2009; Kwon et al., 2016). Arctigenin also strongly inhibited the expression of iNOS (Inducible Nitric Oxide Synthase) and its enzymatic activity (Wang et al., 2007; Zhao et al., 2009). Moreover, it induced endothelial nitric oxide synthase (eNOS) and supressed in a rat model subarachnoid hemorrhage-induced vasospasm by regulation of the PI3K/Akt signaling pathway (Chang et al., 2015). Among the studied phenolic compounds, diarctigenin was found to inhibit the DNA binding ability of NF- $\mathrm{B}$ and to inhibit NF- $\kappa$ B-regulated iNOS expression (Kim et al., 2008), thereby overall targeting NF- $\mathrm{B}$-activating signaling cascade directly to confer anti-inflammatory response. Luteolin, an important flavonoid from burdock was also reported to possess significant anti-inflammatory properties (Ferracane et al., 2010; Nabavi et al., 2015).

\section{Effects Against Skin Conditions}

Leaves of Arctium species have been used in traditional medicinal practices in various skin conditions (e.g., rashes, boils, eczema, ichthyosis, acne, psoriasis, and abscesses) presumably due to the presence of various phenolic compounds. The potent antioxidant and anti-inflammatory properties of these compounds serve to detoxify and mediate healing action (Chan et al., 2011). Several hydroxycinnamic acids which are among the active phytochemicals in the A. lappa extracts (Liu et al., 2012; Tousch et al., 2014) have been found to act as free radical scavengers and possess antioxidant activities, which confer them potential to serve as skin protectors and wound healers (Graf, 1992; Phan et al., 2001; Taofiq et al., 2017). In addition, hydroxycinnamic acid derivatives also display anti-collagenase, anti-inflammatory, antimicrobial and anti-tyrosinase activities, as well as ultraviolet (UV) protective effects, suggesting that they can be exploited as anti-aging and anti-inflammatory agents, preservatives and hyperpigmentationcorrecting ingredients (Ahangarpour et al., 2017). These bioactivities are the reason why burdock extracts find their use in various commercial cosmetic products.

\section{Effect on Potency and Fertility}

Diabetes mellitus induces many complications among which dysfunctions male reproductive system is worth mentioning. Glucose metabolism plays an important regulatory role on the production or development of mature spermatozoa (spermatogenesis) as well as on maintaining specific functions, such as motility and fertilization ability in mature sperm cells. Therefore, it is not surprising that A. lappa root extract, which has hypoglycemic and antioxidative properties, would have beneficial effects on male potency and fertility. Ahangarpour et al. (2015) investigated the effect of A. lappa root extract on gonadotropin, testosterone, and sperm parameters in nicotinamide/streptozotocin-induced diabetic mice (Ahangarpour et al., 2015). The root extract led to increased level of luteinizing hormone $(\mathrm{LH})$, follicle stimulating hormone (FSH), and testosterone as well as enhancement in sperm viability only in diabetic mice compared with the control group, indicating $A$. lappa root extract to be a potentially effective treatment for male sterility arising from diabetic conditions.

\section{Effect on NO Production}

It was reported that arctigenin inhibited NO release by IFN- $\gamma$ signal, whereas it significantly enhanced lipopolysaccharidetriggered NO production in RAW264.7 cells, suggesting that arctigenin may regulate immune responses in activated macrophages and lymphocytes including TNF- $\alpha$ and NO production and lymphocyte proliferation (Cho et al., 1999). Another study shows that arctigenin suppressed the overproduction of NO through down-regulation of iNOS expression and iNOS enzymatic activity in LPS-stimulated macrophage (Zhao et al., 2009). Besides, lappaol F and diarctigenin from Arctium lappa were shown to significantly inhibit NO production in the LPS-stimulated RAW264.7 cells with $\mathrm{IC}_{50}$ values of 9.5 and $9.6 \mu \mathrm{M}$, respectively (Park et al., 2007).

\section{Safety Considerations on Arctium Species}

Several adverse effects have been reported in literature stemming from long-term use of $A$. lappa. For example, contact dermatitis might develop after several days of applying a burdock root plaster to a wound, or even as fast as within $12 \mathrm{~h}$ in some cases (Rodriguez et al., 1995). In one instance, anticholinergic poisoning has been reported upon oral consumption of A. lappa extract (Force, 2001). However, this poisoning later turned out to be caused by products that have been contaminated with root of belladonna (deadly nightshade). The latter herb contains the poisonous chemical atropine. Long-term consumption of burdock also has led to anaphylaxis in one case (Chan et al., 2011). Root oil made from A. lappa was also found to cause unfavorable physiological effects such as redness, and anaphylactic shock (Rodriguez et al., 1995; Lewis and Elvin-Lewis, 2003; Sasaki et al., 2003). Caution is advised for pregnant or nursing women to consume burdock or its extract, as it might have detrimental effects on the fetus (Chan et al., 2011). Burdock can also interfere with blood clotting. People who are already on blood thinning medications are advised not take it without approval from their doctors. Even though burdock is considered a 'safe' 
food, consuming it in large amounts should be avoided due to lack of large amount of safety studies on burdock. More in vivo studies are in particular needed on A. lappa to further evaluate its therapeutic potential and safe application window. Due to the presence of sesquiterpene lactones, the use of Arctium species should be avoided in patients with hypersensitivity to Asteraceae/Compositae (Chan et al., 2011).

\section{SUMMARY}

In summary, the volatile and non-volatile secondary metabolites present in different parts of Arctium species showed pharmacological potential in the treatment of various diseases. The literature existing on extracts of different parts of $A$. lappa and isolated compounds demonstrates antipyretic, antimicrobial, diuretic, diaphoretic, hypoglycaemic, antioxidant, anti-inflammatory, anti-hepatotoxicity, antiulcer, antimutagenicity, and antitumour activities. Hence, Arctium species display a broad therapeutic potential but further studies are needed on potential risks associated with their application.

\section{REFERENCES}

Aboutabl, E., El-Tantawy, M., and Shams, M. (2013). Chemical composition and antimicrobial activity of volatile constituents from the roots, leaves, and seeds of Arctium lappa L. (Asteraceae) grown in Egypt. Egypt. Pharm. J. 12, 173-176. doi: 10.4103/1687-4315.124036

Ahangarpour, A., Heidari, H., Oroojan, A. A., Mirzavandi, F., Nasr Esfehani, K., and Dehghan Mohammadi, Z. (2017). Antidiabetic, hypolipidemic and hepatoprotective effects of Arctium lappa root's hydro-alcoholic extract on nicotinamide-streptozotocin induced type 2 model of diabetes in male mice. Avicenna J. Phytomed. 7, 169-179.

Ahangarpour, A., Oroojan, A. A., Heidari, H., Ghaedi, E., and Taherkhani, R. (2015). Effects of hydro-alcoholic extract from Arctium lappa l.(burdock) root on gonadotropins, testosterone, and sperm count and viability in male mice with nicotinamide/streptozotocin-induced type 2 diabetes. Malays. J. Med. Sci. $22,25-32$.

Al-Shammaa, D. A., Saour, K. Y., and Abdul-Khalik, Z. M. (2017). Phytochemical investigation for the main active constituents in Arctium lappa L. cultivated in Iraq. Iraqi J. Pharm. Sci. 22, 18-24.

Amish Burn Study, G., Kolacz, N. M., Jaroch, M. T., Bear, M. L., and Hess, R. F. (2014). The effect of burns \& wounds (B\&W)/burdock leaf therapy on burninjured amish patients: a pilot study measuring pain levels, infection rates, and healing times. J. Holist. Nurs. 32, 327-340. doi: 10.1177/0898010114525683

Awale, S., Lu, J., Kalauni, S. K., Kurashima, Y., Tezuka, Y., Kadota, S., et al. (2006). Identification of arctigenin as an antitumor agent having the ability to eliminate the tolerance of cancer cells to nutrient starvation. Cancer Res. 66, 1751-1757. doi: 10.1158/0008-5472.can-05-3143

Azizov, U. M., Khadzhieva, U. A., Rakhimov, D. A., Mezhlumyan, L. G., and Salikhov, S. A. (2012). Chemical composition of dry extract of Arctium lappa roots. Chem. Nat. Compd. 47, 1038-1039. doi: 10.1007/s10600-0120142-3

Bai, L., Zhang, H., Liu, Q., Zhao, Y., Cui, X., Guo, S., et al. (2016). Chemical characterization of the main bioactive constituents from fruits of Ziziphus jujuba. Food Funct. 7, 2870-2877. doi: 10.1039/c6fo00613b

Barbosa, J. M., Costa, M., Gomes, C., and Trolin, G. (1993). Isolation of onopordopicrin, the toxic constituent of Arctium-Lappa L. J. Braz. Chem. Soc. 4, 186-187. doi: 10.5935/0103-5053.19930040

Boev, R. S. (2005). A substance with cytostatic and apoptosis-inducing activity from burdock roots. Chem. Sustain. Dev. 13, 119-122.

\section{AUTHOR CONTRIBUTIONS}

All authors listed have made a substantial, direct and intellectual contribution to the work, and approved it for publication.

\section{FUNDING}

This work was supported by the HOMING program of the Foundation for Polish Science co-financed by the European Union under the European Regional Development Fund (Homing/2017-4/41), the Polish KNOW (Leading National Research Centre) Scientific Consortium "Healthy Animal-Safe Food" decision of Ministry of Science and Higher Education No. 05-1/KNOW2/2015, and the Peter und Traudl Engelhorn Foundation for the promotion of Life Sciences.

\section{ACKNOWLEDGMENTS}

We acknowledge Mr. Paul-Marian Szatmari for providing the pictures of Arctium species in Figure 1.

Boldizsar, I., Fuzfai, Z., Toth, F., Sedlak, E., Borsodi, L., and Molnar-Perl, I. (2010). Mass fragmentation study of the trimethylsilyl derivatives of arctiin, matairesinoside, arctigenin, phylligenin, matairesinol, pinoresinol and methylarctigenin: their gas and liquid chromatographic analysis in plant extracts. J. Chromatogr. A 1217, 1674-1682. doi: 10.1016/j.chroma.2010. 01.019

Cao, J., Li, C., Zhang, P., Cao, X., Huang, T., Bai, Y., et al. (2012). Antidiabetic effect of burdock (Arctium lappa L.) root ethanolic extract on streptozotocin-induced diabetic rats. Afr. J. Biotechnol. 11, 9079-9085.

Carlotto, J., de Souza, L. M., Baggio, C. H., Werner, M. F., Maria-Ferreira, D., Sassaki, G. L., et al. (2016). Polysaccharides from Arctium lappa L.: chemical structure and biological activity. Int. J. Biol. Macromol. 91, 954-960. doi: 10. 1016/j.ijbiomac.2016.06.033

Chan, Y. S., Cheng, L. N., Wu, J. H., Chan, E., Kwan, Y. W., Lee, S. M., et al. (2011). A review of the pharmacological effects of Arctium lappa (burdock). Inflammopharmacology 19, 245-254. doi: 10.1007/s10787-010-0062-4

Chang, C.-Z., Wu, S.-C., Chang, C.-M., Lin, C.-L., and Kwan, A.-L. (2015). Arctigenin, a potent ingredient of Arctium lappa L., induces endothelial nitric oxide synthase and attenuates subarachnoid hemorrhage-induced vasospasm through PI3K/Akt pathway in a rat model. Biomed Res. Int. 2015:490209. doi: 10.1155/2015/490209

Charlier, C., and Michaux, C. (2003). Dual inhibition of cyclooxygenase-2 (COX2 ) and 5-lipoxygenase (5-LOX) as a new strategy to provide safer non-steroidal anti-inflammatory drugs. Eur. J. Med. Chem. 38, 645-659. doi: 10.1016/s02235234(03)00115-6

Chen, F. A., Wu, A. B., and Chen, C. Y. (2004). The influence of different treatments on the free radical scavenging activity of burdock and variations of its active components. Food Chem. 86, 479-484. doi: 10.1016/j.foodchem.2003. 09.020

Cho, J. Y., Kim, A. R., Yoo, E. S., Baik, K. U., and Park, M. H. (1999). Immunomodulatory effect of arctigenin, a lignan compound, on tumour necrosis factor-alpha and nitric oxide production, and lymphocyte proliferation. J. Pharm. Pharmacol. 51, 1267-1273. doi: 10.1211/0022 357991777001

da Silva, L. M., Allemand, A., Mendes, D. A. G. B., dos Santos, A. C., Andre, E., de Souza, L. M., et al. (2013). Ethanolic extract of roots from Arctium lappa L. accelerates the healing of acetic acid-induced gastric ulcer in rats: involvement of the antioxidant system. Food Chem. Toxicol. 51, 179-187. doi: 10.1016/j.fct. 2012.09.026 
de Almeida, A. B., Sanchez-Hidalgo, M., Martin, A. R., Luiz-Ferreira, A., Trigo, J. R., Vilegas, W., et al. (2013). Anti-inflammatory intestinal activity of Arctium lappa L. (Asteraceae) in TNBS colitis model. J. Ethnopharmacol. 146, 300-310. doi: 10.1016/j.jep.2012.12.048

de Souza, G. C., Haas, A. P., von Poser, G. L., Schapoval, E. E., and Elisabetsky, E. (2004). Ethnopharmacological studies of antimicrobial remedies in the south of Brazil. J. Ethnopharmacol. 90, 135-143. doi: 10.1016/j.jep.2003. 09.039

Dias, M. M., Zuza, O., Riani, L. R., de Faria Pinto, P., Pinto, P. L. S., Silva, M. P., et al. (2017). In vitro schistosomicidal and antiviral activities of Arctium lappa L.(Asteraceae) against Schistosoma mansoni and Herpes simplex virus-1. Biomed. Pharm. 94, 489-498. doi: 10.1016/j.biopha.2017.07.116

Duh, P. D. (1998). Antioxidant activity of burdock (Arctium lappa Linne): its scavenging effect on free-radical and active oxygen. J. $t$ Am. Oil Chem. Soc. 75, 455-461. doi: 10.1007/s11746-998-0248-8

Dumitrescu, R., Mehedintu, C., Briceag, I., Purcãrea, V. L., and Hudita, D. (2015). Metformin-clinical pharmacology in PCOs. J. Med. Life 8, 187-192.

Eberding, A., Madera, C., Xie, S., Wood, C. A., Brown, P. N., and Guns, E. S. (2007). Evaluation of the antiproliferative effects of Essiac (TM) on in vitro and in vivo models of prostate cancer compared to paclitaxel. Nutr. Cancer Int. J. 58, 188-196. doi: 10.1080/01635580701328396

Erdemoglu, N., Turan, N. N., Akkol, E. K., Sener, B., and Abacioglu, N. (2009). Estimation of anti-inflammatory, antinociceptive and antioxidant activities of Arctium minus (Hill) Bernh. ssp. minus. J. Ethnopharmacol. 121, 318-323. doi: 10.1016/j.jep.2008.11.009

European Medicines Agency (2011). Community Herbal Monograph on Actium lappa L., Radix. EMA/HMPC/246763/2009. Available: https://www.ema.europa.eu/documents/herbal-monograph/final-communityherbal-monograph-arctium-lappa-l-radix_en.pdf (accessed February 15, 2019).

European Scientific Cooperative on Phytotherapy (2003). ESCOP Monographs: The Scientific Foundation for Herbal Medicinal Products. Exeter: European Scientific Cooperative on Phytotherapy.

European Scientific Cooperative on Phytotherapy-The Scientific Foundation for Herbal Medicinal Products (2016). ESCOP Monograph on Arctii radix (Burdock Root). Available: http://escop.com/downloads/arctii/ (accessed February 15, 2019).

Ferracane, R., Graziani, G., Gallo, M., Fogliano, V., and Ritieni, A. (2010). Metabolic profile of the bioactive compounds of burdock (Arctium lappa) seeds, roots and leaves. J. Pharm. Biomed. Anal. 51, 399-404. doi: 10.1016/j.jpba.2009. 03.018

Fierascu, R. C., Georgiev, M. I., Fierascu, I., Ungureanu, C., Avramescu, S. M., Ortan, A., et al. (2018). Mitodepressive, antioxidant, antifungal and anti-inflammatory effects of wild-growing Romanian native Arctium lappa L.(Asteraceae) and Veronica persica Poiret (Plantaginaceae). Food Chem. Toxicol. 111, 44-52. doi: 10.1016/j.fct.2017.11.008

Force, L. H. T. (2001). The Longwood Herbal Task Force. Paris: Task Force.

Fuchigami, M., Kishigami, Y., and Sasaki, A. (1990). Pectic polysaccharides in edible burdock root. J. Home Econ. Jpn. 41, 957-963. doi: 10.11428/jhej1987. 41.957

Gao, H., Li, G., Zhang, J., and Zeng, J. (2008). Arctigenin: a lignan from Arctium lappa. Acta Crystallogr. Sec. E Struct. Rep. Online 64(Pt 8):o1538. doi: 10.1107/ S1600536808021752

Gentil, M., Pereira, J. V., Sousa, Y. T., Pietro, R., Neto, M. D., Vansan, L. P., et al. (2006). In vitro evaluation of the antibacterial activity of Arctium lappa as a phytotherapeutic agent used in intracanal dressings. Phytother. Res. 20, 184-186. doi: 10.1002/ptr.1829

Graf, E. (1992). Antioxidant potential of ferulic acid. Free Radic. Biol. Med. 13, 435-448. doi: 10.1016/0891-5849(92)90184-i

Haghi, G., Hatami, A., and Mehran, M. (2013). UPLC and HPLC of caffeoyl esters in wild and cultivated Arctium lappa L. Food Chem. 138, 321-326. doi: 10.1016/j.foodchem.2012.10.040

Han, B. H., Kang, Y. H., Yang, H. O., and Park, M. K. (1994). A butyrolactone lignan dimer from Arctium-Lappa. Phytochemistry 37, 1161-1163. doi: 10. 1016/s0031-9422(00)89550-3

Han, Z. W., Wang, M. Z., Wang, L. Y., Qu, H., Li, P., and Wang, C. B. (2013). Chemical analysis of burdock root constituents. Asian J. Chem. 25, 2573-2576. doi: 10.14233/ajchem.2013.13468
He, J., Huang, X. Y., Yang, Y. N., Feng, Z. M., Jiang, J. S., and Zhang, P. C. (2016). Two new compounds from the fruits of Arctium lappa. J. Asian Nat. Prod. Res. 18, 423-428. doi: 10.1080/10286020.2016.1145671

Hsieh, C.-J., Kuo, P.-L., Hsu, Y.-C., Huang, Y.-F., Tsai, E.-M., and Hsu, Y.L. (2014). Arctigenin, a dietary phytoestrogen, induces apoptosis of estrogen receptor-negative breast cancer cells through the ROS/p38 MAPK pathway and epigenetic regulation. Free Radic. Biol. Med. 67, 159-170. doi: 10.1016/j. freeradbiomed.2013.10.004

Huang, K., Liang, X. C., Zhong, Y. L., He, W. Y., and Wang, Z. (2015). 5Caffeoylquinic acid decreases diet-induced obesity in rats by modulating PPARalpha and LXRalpha transcription. J. Sci. Food Agric. 95, 1903-1910. doi: $10.1002 /$ jsfa.6896

Huang, X. Y., Feng, Z. M., Yang, Y. N., Jiang, J. S., and Zhang, P. C. (2015). Four new neolignan glucosides from the fruits of Arctium lappa. J. Asian Nat. Prod. Res. 17, 504-511. doi: 10.1080/10286020.2015.1039525

Ichihara, A., Kanai, S., Nakamura, Y., and Sakamura, S. (1978). Structures of Lappaol-F and Lappaol-H, dilignans from Arctium-Lappa L. Tetrahedron Lett. 19, 3035-3038. doi: 10.1016/s0040-4039(01)94932-9

Ichihara, A., Numata, Y., Kanai, S., and Sakamura, S. (1977). New sesquilignans from Arctium-Lappa L - Structure of Lappaol-C, Lappaol-D, and Lappaol-E. Agric. Biol. Chem. 41, 1813-1814. doi: 10.1271/bbb1961.41. 1813

Ichihara, A., Oda, K., Numata, Y., and Sakamura, S. (1976). Lappaol-a and LappaolB, novel lignans from Arctium-Lappa L. Tetrahedron Lett. 17, 3961-3964. doi: 10.1016/s0040-4039(00)92547-4

Ichikawa, K., Kinoshita, T., Nishibe, S., and Sankawa, U. (1986). The Ca2+ antagonist activity of lignans. Chem. Pharm. Bull. 34, 3514-3517. doi: 10.1248/ cpb.34.3514

Ikeda, M., Sato, A., Mochizuki, N., Toyosaki, K., Miyoshi, C., Fujioka, R., et al. (2016). Phase I trial of GBS-01 for advanced pancreatic cancer refractory to gemcitabine. Cancer Sci. 107, 1818-1824. doi: 10.1111/cas. 13086

Jaiswal, R., and Kuhnert, N. (2011). Identification and characterization of five new classes of chlorogenic acids in burdock (Arctium lappa L.) roots by liquid chromatography/tandem mass spectrometry. Food Funct. 2, 63-71. doi: 10. 1039/c0fo00125b

Jaric, S., Popovic, Z., Macukanovic-Jocic, M., Djurdjevic, L., Mijatovic, M., Karadzic, B., et al. (2007). An ethnobotanical study on the usage of wild medicinal herbs from Kopaonik Mountain (Central Serbia). J. Ethnopharmacol. 111, 160-175. doi: 10.1016/j.jep.2006.11.007

Jeelani, S., and Khuroo, M. A. (2012). Triterpenoids from Arctium lappa. Nat. Prod. Res. 26, 654-658. doi: 10.1080/14786419.2010.541886

Kardosova, A., Ebringerova, A., Alfoldi, J., Nosal'ova, G., Franova, S., and Hribalova, V. (2003). A biologically active fructan from the roots of Arctium lappa L., var. Herkules. Int. J. Biol. Macromol. 33, 135-140. doi: 10.1016/S01418130(03)00079-5

Kato, Y., and Watanabe, T. (1993). Isolation and characterization of a xyloglucan from gobo (Arctium-Lappa L). Biosci. Biotechnol. Biochem. 57, 1591-1592. doi: 10.1271/bbb.57.1591

Kim, B. H., Hong, S. S., Kwon, S. W., Lee, H. Y., Sung, H., Lee, I. J., et al. (2008). Diarctigenin, a lignan constituent from Arctium lappa, down-regulated zymosan-induced transcription of inflammatory genes through suppression of DNA binding ability of nuclear factor-kappa B in macrophages. J. Pharmacol. Exp. Ther. 327, 393-401. doi: 10.1124/jpet.108.140145

Kuo, D. H., Hung, M. C., Hung, C. M., Liu, L. M., Chen, F. A., Shieh, P. C., et al. (2012). Body weight management effect of burdock (Arctium lappa L.) root is associated with the activation of AMP-activated protein kinase in human HepG2 cells. Food Chem. 134, 1320-1326. doi: 10.1016/j.foodchem.2012. 03.023

Kwon, K., Koong, H. S., and Kang, K. H. (2016). Effect of burdock extracts upon inflammatory mediator production. Technol. Health Care 24, 459-469. doi: $10.3233 /$ thc- 151123

Lans, C., and Turner, N. (2011). Organic parasite control for poultry and rabbits in British Columbia, Canada. J. Ethnobiol. Ethnomed. 7:21. doi: 10.1186/17464269-7-21

Lans, C., Turner, N., Khan, T., Brauer, G., and Boepple, W. (2007). Ethnoveterinary medicines used for ruminants in British Columbia, Canada. J. Ethnobiol. Ethnomed. 3:11. doi: 10.1186/1746-4269-3-11 
Lee, B. I., Nugroho, A., Bachri, M. S., Choi, J., Lee, K. R., Choi, J. S., et al. (2010). Anti-ulcerogenic effect and HPLC analysis of the caffeoylquinic acidrich extract from Ligularia stenocephala. Biol. Pharm. Bull. 33, 493-497. doi: $10.1248 / \mathrm{bpb} .33 .493$

Lee, J. H., Lee, J. Y., Park, J. H., Jung, H. S., Kim, J. S., Kang, S. S., et al. (2007). Immunoregulatory activity by daucosterol, a beta-sitosterol glycoside, induces protective Th1 immune response against disseminated Candidiasis in mice. Vaccine 25, 3834-3840. doi: 10.1016/j.vaccine.2007.01.108

Lee, J. Y., and Kim, C. J. (2010). Arctigenin, a phenylpropanoid dibenzylbutyrolactone lignan, inhibits type I-IV allergic inflammation and pro-inflammatory enzymes. Arch. Pharm. Res. 33, 947-957. doi: 10.1007/s12272-010-0619-1

Leonard, S. S., Keil, D., Mehlman, T., Proper, S., Shi, X., and Harris, G. K. (2006). Essiac tea: scavenging of reactive oxygen species and effects on DNA damage. J. Ethnopharmacol. 103, 288-296. doi: 10.1016/j.jep.2005.09.013

Lewis, W. H., and Elvin-Lewis, M. P. F. (2003). Medical Botany: Plants Affecting Human Health. Hoboken, NJ: Wiley.

Li, J., Liu, X., Zhou, B., Zhao, J., and Li, S. (2013). Determination of fructooligosaccharides in burdock using HPLC and microwave-assisted extraction. J. Agric. Food Chem. 61, 5888-5892. doi: 10.1021/jf400534n

Lin, L. Z., and Harnly, J. M. (2008). Identification of hydroxycinnamoylquinic acids of arnica flowers and burdock roots using a standardized LC-DADESI/MS profiling method. J. Agric. Food Chem. 56, 10105-10114. doi: 10.1021/ jf802412m

Lin, S. C., Chung, T. C., Lin, C. C., Ueng, T. H., Lin, Y. H., Lin, S. Y., et al. (2000). Hepatoprotective effects of Arctium lappa on carbon tetrachlorideand acetaminophen-induced liver damage. Am. J. Chin. Med. 28, 163-173. doi: 10.1142/S0192415x00000210

Liu, J. Y., Cai, Y. Z., Wong, R. N. S., Lee, C. K. F., Tang, S. C. W., Sze, S. C. W., et al. (2012). Comparative analysis of caffeoylquinic acids and lignans in roots and seeds among various burdock (Arctium lappa) genotypes with high antioxidant activity. J. Agric. Food Chem. 60, 4067-4075. doi: 10.1021/jf2050697

Liu, Q. D., Qin, K. M., Shen, B. J., Cai, H., and Cai, B. C. (2015). Optimization of the processing technology of fructus arctii by response surface methodology. Chin. J. Nat. Med. 13, 222-231. doi: 10.1016/S1875-5364(15)30008-X

Liu, S. M., Chen, K. S., Schliemann, W., and Strack, D. (2005). Isolation and identification of arctiin and arctigenin in leaves of burdock (Arctium lappla L.) by polyamide column chromatography in combination with HPLC-ESI/MS. Phytochem. Anal. 16, 86-89. doi: 10.1002/pca.816

Liu, W., Wang, J. J., Zhang, Z. Z., Xu, J. N., Xie, Z. H., Slavin, M., et al. (2014). In vitro and in vivo antioxidant activity of a fructan from the roots of Arctium lappa L. Int. J. Biol. Macromol. 65, 446-453. doi: 10.1016/j.ijbiomac.2014. 01.062

Lopez-Vinyallonga, S., Arakaki, M., Garcia-Jacas, N., Susanna, A., Gitzendanner, M. A., Soltis, D. E., et al. (2010). Isolation and characterization of novel microsatellite markers for Arctium minus (Compositae). Am. J. Bot. 97, e4-e6. doi: 10.3732/ajb.0900376

Lou, C., Zhu, Z., Zhao, Y., Zhu, R., and Zhao, H. (2017). Arctigenin, a lignan from Arctium lappa L., inhibits metastasis of human breast cancer cells through the downregulation of MMP-2/-9 and heparanase in MDA-MB-231 cells. Oncol. Rep. 37, 179-184. doi: 10.3892/or.2016.5269

Lou, Z. X., Li, C., Kou, X. R., Yu, F. H., Wang, H. X., Smith, G. M., et al. (2016). Antibacterial, antibiofilm effect of burdock (Arctium lappa L.) leaf fraction and its efficiency in meat preservation. J. Food Protect. 79, 1404-1409. doi: 10.4315/0362-028x.J1t-15-576

Lou, Z. X., Wang, H. X., Lv, W. P., Ma, C. Y., Wang, Z. P., and Chen, S. W. (2010a). Assessment of antibacterial activity of fractions from burdock leaf against foodrelated bacteria. Food Control 21, 1272-1278. doi: 10.1016/j.foodcont.2010. 02.016

Lou, Z. X., Wang, H. X., Zhu, S., Zhang, M., Gao, Y., Ma, C. Y., et al. (2010b). Improved extraction and identification by ultra performance liquid chromatography tandem mass spectrometry of phenolic compounds in burdock leaves. J. Chromatogr. A 1217, 2441-2446. doi: 10.1016/j.chroma.2009. 12.022

Machado, F. B., Yamamoto, R. E., Zanoli, K., Nocchi, S. R., Novello, C. R., Schuquel, I. T. A., et al. (2012). Evaluation of the antiproliferative activity of the leaves from Arctium lappa by a bioassay-guided fractionation. Molecules 17, 1852-1859. doi: 10.3390/molecules17021852
Maghsoumi-Norouzabad, L., Alipoor, B., Abed, R., Eftekhar Sadat, B., MesgariAbbasi, M., and Asghari Jafarabadi, M. (2016). Effects of Arctium lappa L.(Burdock) root tea on inflammatory status and oxidative stress in patients with knee osteoarthritis. Int. J. Rheum. Dis. 19, 255-261. doi: 10.1111/1756185X.12477

Maimaitili, A., Shu, Z., Cheng, X., Kaheerman, K., Sikandeer, A., and Li, W. (2017). Arctigenin, a natural lignan compound, induces G0/G1 cell cycle arrest and apoptosis in human glioma cells. Oncol. Lett. 13, 1007-1013. doi: 10.3892/ol. 2016.5474

Maruta, Y., Kawabata, J., and Niki, R. (1995). Antioxidative caffeoylquinic acidderivatives in the roots of burdock (Arctium-Lappa L). J. Agric. Food Chem. 43, 2592-2595. doi: 10.1021/jf00058a007

Matsumoto, T., Hosono-Nishiyama, K., and Yamada, H. (2006). Antiproliferative and apoptotic effects of butyrolactone lignans from Arctium lappa on leukemic cells. Planta Med. 72, 276-278. doi: 10.1055/s-2005-916174

Maxwell, T., Chun, S. Y., Lee, K. S., Kim, S., and Nam, K. S. (2017). The antimetastatic effects of the phytoestrogen arctigenin on human breast cancer cell lines regardless of the status of ER expression. Int. J. Oncol. 50, 727-735. doi: 10.3892/ijo.2016.3825

Miglani, A., and Manchanda, R. K. (2014). Observational study of Arctium lappa in the treatment of acne vulgaris. Homeopathy 103, 203-207. doi: 10.1016/j.homp. 2013.12.002

Milani, E., Koocheki, A., and Golimovahhed, Q. A. (2011). Extraction of inulin from Burdock root (Arctium lappa) using high intensity ultrasound. Int. J. Food Sci. Technol. 46, 1699-1704. doi: 10.1111/j.1365-2621.2011.02673.x

Ming, D. S., Guns, E. S., Eberding, A., and Towers, G. H. N. (2004). Isolation and characterization of compounds with anti-prostate cancer activity from Arictium lappa L. using bioactivity-guided fractionation. Pharm. Biol. 42, 44-48. doi: 10.1080/13880200490505474

Miyazawa, M., Yagi, N., and Taguchi, K. (2005). Inhibitory compounds of \&alpha;glucosidase activity from Arctium lappa L. J. Oleo Sci. 54, 589-594. doi: 10.5650/ jos.54.589

Mkrtchian, T. A., Snapian, G. G., and Nikogosian, G. A. (1998). Pectin production from burdock (Arctium sp.). Ukr Biokhim Zh 70, 98-105.

Mosaddegh, M., Naghibi, F., Moazzeni, H., Pirani, A., and Esmaeili, S. (2012). Ethnobotanical survey of herbal remedies traditionally used in Kohghiluyeh va Boyer Ahmad province of Iran. J. Ethnopharmacol. 141, 80-95. doi: 10.1016/j. jep.2012.02.004

Nabavi, S. F., Braidy, N., Gortzi, O., Sobarzo-Sanchez, E., Daglia, M., Skalicka-Woźniak, K., et al. (2015). Luteolin as an anti-inflammatory and neuroprotective agent: a brief review. Brain Res. Bull. 119, 1-11. doi: 10.1016/j. brainresbull.2015.09.002

Neves, J. M., Matos, C., Moutinho, C., Queiroz, G., and Gomes, L. R. (2009). Ethnopharmacological notes about ancient uses of medicinal plants in Tras-osMontes (northern of Portugal). J. Ethnopharmacol. 124, 270-283. doi: 10.1016/ j.jep.2009.04.041

Olennikov, D. N., and Tankhaeva, L. M. (2011). A quantitative assay for total fructans in burdock (Arctium spp.) roots. Russ. J. Bioorg. Chem. 37, 893-898. doi: 10.1134/S1068162011070181

Park, S. Y., Hong, S. S., Han, X. H., Hwang, J. S., Lee, D., Ro, J. S., et al. (2007). Lignans from Arctium lappa and their inhibition of LPS-induced nitric oxide production. Chem. Pharm. Bull. 55, 150-152. doi: 10.1248/cpb.55.150

Pereira, J. V., Bergamo, D. C. B., Pereira, J. O., França, S. D. C., Pietro, R. C. L. R., and Silva-Sousa, Y. T. C. (2005). Antimicrobial activity of Arctium lappa constituents against microorganisms commonly found in endodontic infections. Braz. Dent. J. 16, 192-196. doi: 10.1590/s0103-6440200500030 0004

Phan, T.-T., Wang, L., See, P., Grayer, R. J., Chan, S.-Y., and Lee, S. T. (2001). Phenolic compounds of Chromolaena odorata protect cultured skin cells from oxidative damage: implication for cutaneous wound healing. Biol. Pharm. Bull. 24, 1373-1379. doi: 10.1248/bpb.24.1373

Pieroni, A., Giusti, M. E., and Quave, C. L. (2011). Cross-cultural ethnobiology in the western balkans: medical ethnobotany and ethnozoology among albanians and serbs in the Pešter Plateau, Sandžak, South-Western Serbia. Hum. Ecol. 39, 333-349. doi: 10.1007/s10745-011-9401-3

Predes, F. S., Ruiz, A. L. T. G., Carvalho, J. E., Foglio, M. A., and Dolder, H. (2011). Antioxidative and in vitro antiproliferative activity of Arctium lappa root extracts. BMC Compl. Altern. Med. 11:25. doi: 10.1186/1472-6882-11-25 
Qin, K., Liu, Q., Cai, H., Cao, G., Lu, T., Shen, B., et al. (2014). Chemical analysis of raw and processed fructus arctii by high-performance liquid chromatography/diode array detection-electrospray ionization-mass spectrometry. Pharmacogn. Mag. 10, 541-546. doi: 10.4103/0973-1296.14 1806

Rajasekharan, S. K., Ramesh, S., Bakkiyaraj, D., Elangomathavan, R., and Kamalanathan, C. (2015). Burdock root extracts limit quorum-sensingcontrolled phenotypes and biofilm architecture in major urinary tract pathogens. Urolithiasis 43, 29-40. doi: 10.1007/s00240-014-0720-x

Rodriguez, P., Blanco, J., Juste, S., Garces, M., Perez, R., Alonso, L., et al. (1995). Allergic contact-dermatitis due to burdock (Arctium-Lappa). Contact Dermat. 33, 134-135. doi: 10.1111/j.1600-0536.1995.tb00524.x

Saleh, N. A. M., and Bohm, B. A. (1971). Flavonoids of arctium-minus (Compositae). Experientia 27:1494. doi: 10.1007/Bf02154314

Saric-Kundalic, B., Dobes, C., Klatte-Asselmeyer, V., and Saukel, J. (2010). Ethnobotanical study on medicinal use of wild and cultivated plants in middle, south and west Bosnia and Herzegovina. J. Ethnopharmacol. 131, 33-55. doi: 10.1016/j.jep.2010.05.061

Sasaki, Y., Kimura, Y., Tsunoda, T., and Tagami, H. (2003). Anaphylaxis due to burdock. Int. J. Dermatol. 42, 472-473. doi: 10.1046/j.1365-4362.2003.0171 6_2.x

Savina, A. A., Sheichenko, V. I., Stikhin, Y. V., Stikhin, V. A., Sokol'skaya, T. A., Anisimova, O. S., et al. (2006). Sesquiterpene lactones in juice of great burdock leaves. Pharm. Chem. J. 40, 624-626. doi: 10.1007/s11094-006-02 07-3

Schroder, H. C., Merz, H., Steffen, R., Muller, W. E., Sarin, P. S., Trumm, S., et al. (1990). Differential in vitro anti-HIV activity of natural lignans. Z Naturforsch C 45, 1215-1221. doi: 10.1515/znc-1990-11-1222

Sezik, E., Yesilada, E., Honda, G., Takaishi, Y., Takeda, Y., and Tanaka, T. (2001). Traditional medicine in Turkey X. folk medicine in central anatolia. J. Ethnopharmacol. 75, 95-115. doi: 10.1016/s0378-8741(00) 00399-8

Sezik, E., Yesilada, E., Shadidoyatov, H., Kulivey, Z., Nigmatullaev, A. M., Aripov, H. N., et al. (2004). Folk medicine in Uzbekistan. I. Toshkent, Djizzax, and Samarqand provinces. J. Ethnopharmacol. 92, 197-207. doi: 10.1016/j.jep.2004. 02.016

Su, S., Cheng, X. L., and Wink, M. (2015). Natural lignans from Arctium lappa modulate P-glycoprotein efflux function in multidrug resistant cancer cells. Phytomedicine 22, 301-307. doi: 10.1016/j.phymed.2014.12.009

Sun, Q., Liu, K., Shen, X., Jin, W., Jiang, L., Sheikh, M. S., et al. (2014). Lappaol F, a novel anticancer agent isolated from plant Arctium Lappa L. Mol. Cancer Ther. 13, 49-59. doi: 10.1158/1535-7163.MCT-13-0552

Takasugi, M., Kawashima, S., Katsui, N., and Shirata, A. (1987). Studies on stress metabolites.5. 2 polyacetylenic phytoalexins from Arctium-Lappa. Phytochemistry 26, 2957-2958. doi: 10.1016/S0031-9422(00)84570-7

Tamayo, C., Richardson, M. A., Diamond, S., and Skoda, I. (2000). The chemistry and biological activity of herbs used in Flor-Essence (TM) herbal tonic and Essiac (TM). Phytother. Res. 14, 1-14. doi: 10.1002/(sici)1099-1573(200002)14: $1<1:$ :aid-ptr580 $>3.3 . c 0 ; 2-\mathrm{f}$

Tang, Y. X., Lou, Z. X., Rahman, M. R. T., Al-Hajj, N. Q., and Wang, H. X. (2014). Chemical composition and anti-biofilm activity of burdock (Arctium lappa $\mathrm{L}$ Asteraceae) leaf fractions against Staphylococcus aureus. Trop. J. Pharm. Res. 13, 1933-1939. doi: 10.4314/tjpr.v13i11.23

Taofiq, O., González-Paramás, A. M., Barreiro, M. F., and Ferreira, I. C. (2017). Hydroxycinnamic acids and their derivatives: cosmeceutical significance, challenges and future perspectives, a review. Molecules 22, 281-304. doi: 10. 3390/molecules22020281

Tardio, J., Pascual, H., and Morales, R. (2005). Wild food plants traditionally used in the province of Madrid, central Spain. Econ. Bot. 59, 122-136. doi: 10.1663/0013-0001(2005)059\%5B0122:wfptui\%5D2.0.co;2

Ting-Guo, K., Wen-Jun, Z., Hitoshi, T., Tomoko, K., Zhao-Hui, X. U., SongSong, Y., et al. (2001). Pharmacognostical evaluation of arctii fructus (4): chemical constituents from fruits ofarctium tomentosum(Natural Medicine Note). 生葲學雜声. Nat. Med. 55:153.

Tousch, D., Bidel, L. P. R., Cazals, G., Ferrare, K., Leroy, J., Faucanie, M., et al. (2014). Chemical analysis and antihyperglycemic activity of an original extract from burdock root (Arctium lappa). J. Agric. Food Chem. 62, 7738-7745. doi: $10.1021 /$ jf500926v
Turdumambetov, K., Bakirov, G. A., and Rakhimov, D. A. (2004). Glucofructans from arctium tomentosum roots. Chem. Nat. Compd. 40, 211-214. doi: 10.1023/ B:CONC.0000039125.29910.a1

Uchiyama, Y., Tagami, J., Kamisuki, S., Kasai, N., Oshige, M., Chiku, H., et al. (2005). Selective inhibitors of terminal deoxyribonucleotidyltransferase (TdT): baicalin and genistin. Biochim. Biophys. Acta 1725, 298-304. doi: 10.1016/j. bbagen.2005.06.017

Umehara, K., Sugawa, A., Kuroyanagi, M., Ueno, A., and Taki, T. (1993). Studies on differentiation-inducers from arctium-fructus. Chem. Pharm. Bull. 41, 1774-1779. doi: 10.1248/cpb.41.1774

Wang, B. S., Yen, G. C., Chang, L. W., Yen, W. J., and Duh, P. D. (2007). Protective effects of burdock (Arctium lappa Linne) on oxidation of low-density lipoprotein and oxidative stress in RAW 264.7 macrophages. Food Chem. 101, 729-738. doi: 10.1016/j.foodchem.2006.01.051

Wang, H. Y., and Yang, J. S. (1993). Studies on the chemical constituents of Arctium lappa L. Yao Xue Xue Bao 28, 911-917.

Wang, H. Y., and Yang, J. S. (1995). Neoarctin A from Arctium lappa L. Chin. Chem. Lett. 6, 217-220.

Wang, R., Ayano, H., Furumoto, T., Kondo, A., and Fukui, H. (2001). Variation of the content of chlorogenic acid derivatives among cultivars and market items of burdock (Arctium lappa L.). Nippon Shokuhin Kagaku Kogaku Kaishi 48, 857-862. doi: 10.3136/nskkk.48.857

Wang, X., Cheng, C., Yang, Y., and Zheng, C. (2004). Analysis of the essential oil from Arctium lappa L. Nat. Prod. Res. Dev. 16, 33-35.

Wang, X., Li, F. W., Sun, Q. L., Yuan, J. P., Jiang, T., and Zheng, C. C. (2005). Application of preparative high-speed counter-current chromatography for separation and purification of arctiin from Fructus Arctii. J. Chromatogr. A 1063, 247-251. doi: 10.1016/j.chroma.2004.11.077

Wang, Y., Zhang, N., Kan, J., Zhang, X., Wu, X., Sun, R., et al. (2019). Structural characterization of water-soluble polysaccharide from Arctium lappa and its effects on colitis mice. Carbohydr. Polym. 213, 89-99. doi: 10.1016/j.carbpol. 2019.02.090

Washino, T., Kobayashi, H., and Ikawa, Y. (1987). Structures of Lappaphen-a and Lappaphen-B, new guaianolides linked with a sulfur-containing acetyleniccompound, from Arctium-Lappa-L. Agric. Biol. Chem. 51, 1475-1480. doi: 10.1271/bbb1961.51.1475

Washino, T., Yoshikura, M., and Obata, S. (1986). New sulfur-containing acetylenic-compounds from Arctium-Lappa. Agric. a Biol. Chem. 50, 263-269. doi: 10.1271/bbb1961.50.263

Wu, Y.-C., Lin, L.-F., Yeh, C.-S., Lin, Y.-L., Chang, H.-J., Lin, S.-R., et al. (2010). Burdock essence promotes gastrointestinal mucosal repair in ulcer patients. Fooyin J. Health Sci. 2, 26-31. doi: 10.1016/S1877-8607(10)60010-0

Xu, Z. H., Gu, C. C., Wang, K., Ju, J. X., Wang, H. Y., Ruan, K. F., et al. (2015). Arctigenic acid, the key substance responsible for the hypoglycemic activity of Fructus Arctii. Phytomedicine 22, 128-137. doi: 10.1016/j.phymed.2014. 11.006

Xu, Z. H., Ju, J. X., Wang, K., Gu, C. C., and Feng, Y. (2014). Evaluation of hypoglycemic activity of total lignans from Fructus Arctii in the spontaneously diabetic Goto-Kakizaki rats. J. Ethnopharmacol. 151, 548-555. doi: 10.1016/j. jep.2013.11.021

Yang, Y. N., Huang, X. Y., Feng, Z. M., Jiang, J. S., and Zhang, P. C. (2015). New butyrolactone type lignans from arctii fructus and their antiinflammatory activities. J. Agric. Food Chem. 63, 7958-7966. doi: 10.1021/acs.jafc.5b02838

Yang, Y. N., Zhang, F., Feng, Z. M., Jiang, J. S., and Zhang, P. C. (2012). Two new neolignan glucosides from Arctii Fructus. J. Asian Nat. Prod. Res. 14, 981-985. doi: 10.1080/10286020.2012.729050

Yayli, N., Yasar, A., Gulec, C., Usta, A., Kolayli, S., Coskuncelebi, K., et al. (2005). Composition and antimicrobial activity of essential oils from Centaurea sessilis and Centaurea armena. Phytochemistry 66, 1741-1745. doi: 10.1016/j.phytochem.2005.04.006

Yong, M., Kun, G., and Qiu, M. H. (2007). A new lignan from the seeds of Arctium lappa. J. Asian Nat. Prod. Res. 9, 541-544. doi: 10.1080/10286020600882577

Yu, B. S., Yan, X. P., Xiong, J. Y., and Xin, Q. (2003). Simultaneous determination of chlorogenic acid, forsythin and arctiin in Chinese traditional medicines preparation by reversed phase-HPLC. Chem. Pharm. Bull. 51, 421-424. doi: $10.1248 / \mathrm{cpb} .51 .421$

Zhao, C., She, T., Wang, L., Su, Y., Qu, L., Gao, Y., et al. (2015). Daucosterol inhibits cancer cell proliferation by inducing autophagy through reactive 
oxygen species-dependent manner. Life Sci. 137, 37-43. doi: 10.1016/j.lfs.2015. 07.019

Zhao, F., Wang, L., and Liu, K. (2009). In vitro anti-inflammatory effects of arctigenin, a lignan from Arctium lappa L., through inhibition on iNOS pathway. J. Ethnopharmacol. 122, 457-462. doi: 10.1016/j.jep.2009.01.038

Zhao, J. L., Evangelopoulos, D., Bhakta, S., Gray, A. I., and Seidel, V. (2014). Antitubercular activity of Arctium lappa and Tussilago farfara extracts and constituents. J. Ethnopharmacol. 155, 796-800. doi: 10.1016/j.jep.2014.06.034

Zhao, Y., and Zhou, X. (2015). “Arctium lappa L. 牛蒡子 (Niubangzi, Great Burdock)," in Dietary Chinese Herbs: Chemistry, Pharmacology and Clinical Evidence, eds Y. Liu, Z. Wang, and J. Zhang (Vienna: Springer Vienna), 301-305. doi: 10.1007/978-3-211-99448-1_33

Zhou, X., Zhang, H., Ge, L., Gong, H., and Tian, S. (2011). Determination of arctiin and arctigenin contents in arctium tomentosum mill. by HPLC method. E J. Chem. 8, S372-S376. doi: 10.1155/2011/ 517681

Zimmermann, S., Thomi, S., Kaiser, M., Hamburger, M., and Adams, M. (2012). Screening and HPLC-based activity profiling for new antiprotozoal leads from european plants. Sci. Pharm. 80, 205-213. doi: 10.3797/scipharm. 1111-13

Zong, G., Zhu, J., Sun, L., Ye, X., Lu, L., Jin, Q., et al. (2013). Associations of erythrocyte fatty acids in the de novo lipogenesis pathway with risk of metabolic syndrome in a cohort study of middle-aged and older Chinese. Am. J. Clin. Nutr. 98, 319-326. doi: 10.3945/ajcn.113.061218

Conflict of Interest Statement: The authors declare that the research was conducted in the absence of any commercial or financial relationships that could be construed as a potential conflict of interest.

Copyright (C) 2019 Wang, Bădărau, Swamy, Shaw, Maggi, da Silva, López, Yeung, Mocan and Atanasov. This is an open-access article distributed under the terms of the Creative Commons Attribution License (CC BY). The use, distribution or reproduction in other forums is permitted, provided the original author(s) and the copyright owner(s) are credited and that the original publication in this journal is cited, in accordance with accepted academic practice. No use, distribution or reproduction is permitted which does not comply with these terms. 